\title{
Transverse linear dynamics in an axisymmetric ionization cooling channel
}

\author{
G. Dugan \\ Laboratory of Nuclear Studies, Cornell University, Ithaca, New York 14853
}

(Received 29 April 2001; published 3 October 2001)

\begin{abstract}
This paper outlines a formalism for the description of the linear transverse dynamics of charged particles in an axisymmetric ionization cooling channel. The particle trajectories in the absence of Coulomb scattering are described in terms of lattice functions à la Courant and Snyder, which depend only on the electric and magnetic fields in the channel. The process of multiple Coulomb scattering, which introduces stochastic terms into the particle equations of motion, is treated (in Gaussian approximation) by obtaining the distribution function in phase space as a solution of a Fokker-Planck equation. The distribution function is then used to obtain moment equations for the transverse variables and for combinations of variables such as the emittance and angular momentum. The distribution function is also used to obtain an expression for the peak four-dimensional phase space density and for the fraction of the beam that is within a certain area in phase space. The special case of a periodic channel is then considered and expressions for the asymptotic rms emittance and peak phase space density are obtained. Finally, the application of the general formalism to a numerical example, based on the reported design of a cooling channel for a neutrino source, is considered, and comparisons are made with numerical simulations of that channel.
\end{abstract}

DOI: 10.1103/PhysRevSTAB.4.104001

PACS numbers: 29.27.-a, 41.75.-i, 41.85.-p

\section{INTRODUCTION}

The motion of beams of charged particles in axisymmetric magnetic and electric fields has been the subject of considerable study, in connection with the ionization cooling of muons [1,2]. Most of the work to date on ionization cooling has involved numerical simulations, which have the virtue of being able to include both linear and nonlinear dynamics, and can treat the complex interplay between the longitudinal and transverse dynamics present in any ionization cooling channel.

Despite the advantages of numerical simulations, there is also a need for analytical descriptions of ionization cooling systems. Analytical treatments can provide a useful check on, and complement to, numerical work. Because they provide a different view, they also may allow the development of physical intuition about a system in ways that are not possible with numerical simulations.

The description of the linear dynamics of a system forms the basic building block upon which subsequent work, such as the study of nonlinear dynamics, must be founded. In the case of ionization cooling in a solenoidal channel, the transverse linear dynamics in four-dimensional phase space are, in many ways, simpler than the longitudinal dynamics, despite having two more degrees of freedom. This simplicity is related to the axial symmetry of the electric and magnetic fields in a cooling channel. Consequently, it is reasonable to begin with an analytical description of the transverse linear dynamics, despite the fact that many of the real performance challenges are related to the longitudinal dynamics. Several authors [3-5] have, in fact, made considerable progress in developing analytical descriptions of transverse ionization cooling in the linear approxima- tion. The work described in this paper is an extension of, and benefits from, this prior work. To the extent possible, we have used the same notation as in Ref. [3], for ease of comparison with that paper.

In this paper, we start in Sec. II with a development of the equations of motion for a charged particle in axisymmetric static magnetic and rf electromagnetic fields, such as in a solenoidal ionization cooling channel. Ionization cooling requires the placement of media in the path of the particle to provide energy loss; longitudinal momentum is restored by the axial rf electric field, and the net result is a reduction in the transverse emittance. The presence of the media, however, inevitably introduces stochastic emittance growth due to multiple Coulomb scattering, which limits the cooling performance. In Sec. III, we introduce the effects of the media into the equations of motion: the equations become stochastic differential equations.

In Sec. IV, we make several approximations, such as the paraxial approximation, which linearize the stochastic equations of motion. In Sec. V, we solve the linear equations of motion without the stochastic terms, in terms of lattice functions similar to those in the standard CourantSnyder [6] formalism. In Sec. VI, we treat the stochastic terms describing the multiple Coulomb scattering by introducing and solving a Fokker-Planck equation for the distribution function in four-dimensional phase space. The equation is solved by a method [7] originally developed for the treatment of Brownian motion (see also Ref. [5]).

In Sec. VII, we use the solution to derive moment equations for the new variables, and in Sec. VIII we obtain the distribution function and moment equations in the usual phase space variables. In Sec. IX, we consider a collection of particles forming a beam, described by an initial 
distribution. Relations are obtained for the lattice function matching requirements of this beam into the cooling channel. Expressions for the rms emittance and angular momentum of the beam, as it is transported down the cooling channel, are obtained.

In Sec. X, for the case of a Gaussian initial distribution, the distribution function in four-dimensional phase space, as a function of distance along the cooling channel, is given. An expression for the distribution function in the four-dimensional emittance is also derived. In Sec. XI, we consider the application of the general formalism to the case of periodic axisymmetric channels and derive expressions for the mean number of periods for damping and the asymptotic values of the rms emittance, angular momentum, and peak phase space density.

In Sec. XII, some of the results from the formalism described here are compared with prior work [3]. In Sec. XIII, we consider the application of the general formalism to a numerical example based on the design of the ionization cooling channel for the Fermilab neutrino source [8] study. The lattice functions are plotted, and the evolution of the rms emittance, angular momentum, and beam distribution function down the cooling channel are displayed and compared with numerical simulations [8]. Finally, we present a summary in Sec. XIV.

In what follows, we start from first principles, and considerable detail is presented in the derivations, in order to make the assumptions clear. The result of this is a somewhat lengthy work. Nevertheless, the advantage of clarity was felt by the author to outweigh the disadvantage of lack of brevity.

\section{EQUATIONS OF MOTION FOR PARTICLES IN AXISYMMETRIC STATIC MAGNETIC AND RF ELECTROMAGNETIC FIELDS}

We start with the Hamiltonian for a charged particle of mass $m$ and charge $e$ moving under the influence of a static magnetic field and an rf electromagnetic field. The particle's motion will be described in a right-handed Cartesian coordinate system $(x, y, s)$. The transverse coordinates are $x$ and $y$, the longitudinal coordinate is $s$. The electromagnetic potentials are $\mathbf{A}(x, y, s, t)$ and $V(x, y, s, t)$ and the fields are given by $\mathbf{B}=\nabla \times \mathbf{A}$ and $\mathbf{E}=-\nabla V-\frac{\partial \mathbf{A}}{\partial t}$. The Hamiltonian is

$$
\begin{aligned}
& H\left(x, y, s, p_{x}, p_{y}, p_{s}, t\right) \\
& \quad=e V(x, y, s, t)+c \sqrt{\left[p_{x}-e A_{x}(x, y, s, t)\right]^{2}+\left[p_{y}-e A_{y}(x, y, s, t)\right]^{2}+\left[p_{s}-e A_{s}(x, y, s, t)\right]^{2}+(m c)^{2}},
\end{aligned}
$$

in which $\mathbf{p}$ is the canonical momentum. The components of the mechanical momentum $\mathbf{P}$ are given by

$$
\begin{gathered}
P_{x}=m \gamma \nu_{x}=p_{x}-e A_{x}(x, y, s, t), \quad P_{y}=m \gamma \nu_{y}=p_{y}-e A_{y}(x, y, s, t), \\
P_{s}=m \gamma \nu_{s}=p_{s}-e A_{s}(x, y, s, t) .
\end{gathered}
$$

As usual, $\gamma=\frac{\mathcal{E}}{m c^{2}}$, where $\mathcal{E}$ is the total energy, and $\mathbf{v}$ is the velocity. In what follows, dots will represent differentiation with respect to time, and primes will represent differentiation with respect to $s$. Let $l$ represent the distance that the particle travels along its actual path. Its rate of change with $s$,

$$
\frac{d l}{d s}=l^{\prime}=\frac{\nu}{\dot{s}}=\sqrt{1+x^{\prime 2}+y^{\prime 2}} .
$$

The particle's velocity components can be written in terms of $l^{\prime}$ as

$$
\dot{s}=\nu_{s}=\frac{\nu}{l^{\prime}}, \quad \dot{x}=x^{\prime} \dot{s}=x^{\prime} \frac{\nu}{l^{\prime}}, \quad \dot{y}=y^{\prime} \dot{s}=y^{\prime} \frac{\nu}{l^{\prime}} .
$$

Hamilton's equations give the equations of motion:

$$
\begin{aligned}
& \frac{d p_{x}}{d s}=p_{x}^{\prime}=-\frac{1}{\dot{s}} \frac{\partial H}{\partial x}=e\left(\frac{\partial A_{s}}{\partial x}+x^{\prime} \frac{\partial A_{x}}{\partial x}+y^{\prime} \frac{\partial A_{y}}{\partial x}-\frac{l^{\prime}}{\nu} \frac{\partial V}{\partial x}\right), \\
& \frac{d p_{y}}{d s}=p_{y}^{\prime}=-\frac{1}{\dot{s}} \frac{\partial H}{\partial y}=e\left(\frac{\partial A_{s}}{\partial y}+x^{\prime} \frac{\partial A_{x}}{\partial y}+y^{\prime} \frac{\partial A_{y}}{\partial y}-\frac{l^{\prime}}{\nu} \frac{\partial V}{\partial y}\right), \\
& \frac{d p_{s}}{d s}=p_{s}^{\prime}=-\frac{1}{\dot{s}} \frac{\partial H}{\partial s}=e\left(\frac{\partial A_{s}}{\partial s}+x^{\prime} \frac{\partial A_{x}}{\partial s}+y^{\prime} \frac{\partial A_{y}}{\partial s}-\frac{l^{\prime}}{\nu} \frac{\partial V}{\partial s}\right) .
\end{aligned}
$$




\section{INCLUSION OF THE EFFECTS OF A MEDIUM ON THE EQUATIONS OF MOTION}

We now add the effects of the medium in which the particle travels: ionization energy loss and multiple Coulomb scattering. Ionization energy loss is in fact a stochastic process, but the fluctuations are not important for a linear analysis of the transverse dynamics. We will thus consider only the average energy loss, whose effect over a path length $\Delta l$ is to produce a reduction in the total energy:

$$
\Delta \mathcal{E}=-\frac{d \mathcal{E}}{d l} \Delta l
$$

We will also neglect the dependence of $\frac{d \mathcal{E}}{d l}$ on energy. The corresponding change in the mechanical momentum is

$$
\begin{aligned}
\Delta \mathbf{P} & =\frac{\mathbf{P}}{\beta^{2} \mathcal{E}} \Delta \mathcal{E}=-\frac{\mathbf{P}}{\beta^{2} \mathcal{E}} \frac{d \mathcal{E}}{d l} \Delta l \\
& =-\frac{1}{\beta c P} \frac{d \mathcal{E}}{d l} \Delta l\left(P_{s} \hat{\mathbf{s}}+P_{x} \hat{\mathbf{x}}+P_{y} \hat{\mathbf{y}}\right) .
\end{aligned}
$$

Then the rate of change in the canonical momentum is

$$
\begin{aligned}
\frac{d \mathbf{p}}{d s} & =-\frac{1}{P \beta c} \frac{d \mathcal{E}}{d l} l^{\prime}\left(P_{s} \hat{\mathbf{s}}+P_{x} \hat{\mathbf{x}}+P_{y} \hat{\mathbf{y}}\right) \\
& =-\frac{1}{\beta c} \frac{d \mathcal{E}}{d l}\left(\hat{\mathbf{s}}+x^{\prime} \hat{\mathbf{x}}+y^{\prime} \hat{\mathbf{y}}\right)
\end{aligned}
$$

These terms should be added to Eq. (2.5) to include the effect of ionization energy loss.

Multiple Coulomb scattering causes a stochastic change in the trajectory angles:

$$
\frac{d x^{\prime}}{d l}=G(l) \xi_{x}(l), \quad \frac{d y^{\prime}}{d l}=G(l) \xi_{y}(l)
$$

in which $G(l)$ is related to the mean square multiple scattering angle. $\xi_{x, y}(l)$ are uncorrelated unit noise functions, having the properties

$$
\overline{\xi_{i}(l)}=0, \quad \overline{\xi_{i}(l) \xi_{j}\left(l^{\prime}\right)}=\delta_{i j} \delta\left(l-l^{\prime}\right),
$$

in which $i$ and $j$ refer to $x$ and $y$. The average here (denoted by the overbar) refers to an average over the ensemble of possible realizations of the noise.
It will be useful for later work to introduce the rate of change of the mean square transverse angle due to multiple Coulomb scattering. The total integrated change in $x^{\prime}$ over a distance $l$, due to multiple Coulomb scattering, is

$$
x^{\prime}(l)=\int_{0}^{l} d l^{\prime} G\left(l^{\prime}\right) \xi_{x}\left(l^{\prime}\right)
$$

The average change in $x^{\prime}$, and the average of the square of the change in $x^{\prime}$, over the noise ensemble, are

$$
\begin{aligned}
\overline{x^{\prime}} & =\int_{0}^{l} d l^{\prime} G\left(l^{\prime}\right) \overline{\xi_{x}\left(l^{\prime}\right)}=0 \\
\overline{\left(x^{\prime}\right)^{2}} & =\int_{0}^{l} d l^{\prime} G\left(l^{\prime}\right) \int_{0}^{l} d l^{\prime \prime} G\left(l^{\prime \prime}\right) \overline{\xi_{x}\left(l^{\prime}\right) \xi_{x}\left(l^{\prime \prime}\right)} \\
& =\int_{0}^{l} d l^{\prime} G\left(l^{\prime}\right)^{2},
\end{aligned}
$$

using Eq. (3.5). The rate of change of the mean square angle is

$$
\frac{d \overline{\left(x^{\prime}\right)^{2}}}{d l}=G(l)^{2}=\overline{\theta^{2}} n(l)
$$

in which $n(l)$ is the number of multiple Coulomb scattering events per unit distance and $\overline{\theta^{2}}$ is the mean square projected (plane) scattering angle per event. The standard relation [9] for multiple Coulomb scattering in the Moliere approximation gives

$$
G(l)^{2}=n(l) \overline{\theta^{2}} \approx\left(\frac{13.6 \mathrm{MeV}}{\beta c P}\right)^{2} \frac{I}{L_{\mathrm{rad}}},
$$

in which $L_{\mathrm{rad}}$ is the radiation length in the material.

The changes in the canonical momenta can then be represented as

$$
\begin{aligned}
\frac{d p_{x}}{d s} & =m \gamma l^{\prime} \frac{d \dot{x}}{d l}=m \gamma \nu \frac{d x^{\prime}}{d l}=P G \xi_{x}, \\
\frac{d p_{y}}{d s} & =P G \xi_{y} .
\end{aligned}
$$

Introducing the effects of the medium [Eqs. (3.3) and (3.10)] into Eq. (2.5) then gives

$$
\begin{aligned}
& p_{s}^{\prime}=e\left(\frac{\partial A_{s}}{\partial s}+x^{\prime} \frac{\partial A_{x}}{\partial s}+y^{\prime} \frac{\partial A_{y}}{\partial s}-\frac{l^{\prime}}{\nu} \frac{\partial V}{\partial s}\right)-\frac{1}{\beta c} \frac{d \mathcal{E}}{d l}, \\
& p_{x}^{\prime}=e\left(\frac{\partial A_{s}}{\partial x}+x^{\prime} \frac{\partial A_{x}}{\partial x}+y^{\prime} \frac{\partial A_{y}}{\partial x}-\frac{l^{\prime}}{\nu} \frac{\partial V}{\partial x}\right)-\frac{1}{\beta c} \frac{d \mathcal{E}}{d l} x^{\prime}+P G \xi_{x}, \\
& p_{y}^{\prime}=e\left(\frac{\partial A_{s}}{\partial y}+x^{\prime} \frac{\partial A_{x}}{\partial y}+y^{\prime} \frac{\partial A_{y}}{\partial y}-\frac{l^{\prime}}{\nu} \frac{\partial V}{\partial y}\right)-\frac{1}{\beta c} \frac{d \mathcal{E}}{d l} y^{\prime}+P G \xi_{y} .
\end{aligned}
$$

To obtain equations of motion, we rewrite Eq. (2.2), introducing $P_{s}$ into the first two equations,

$$
P_{s} x^{\prime}=p_{x}-e A_{x}(x, y, s, t), \quad P_{s} y^{\prime}=p_{y}-e A_{y}(x, y, s, t), \quad P_{s}=p_{s}-e A_{s}(x, y, s, t) .
$$


We differentiate Eq. (3.12) once with respect to $s$. The result may be used to eliminate the canonical momenta in Eq. (3.11), obtaining

$$
\begin{aligned}
P_{s}^{\prime} x^{\prime}+P_{s} x^{\prime \prime} & =-e\left[\frac{\partial A_{x}}{\partial s}-\frac{\partial A_{s}}{\partial x}+y^{\prime}\left(\frac{\partial A_{x}}{\partial y}-\frac{\partial A_{y}}{\partial x}\right)+\frac{m \gamma}{P_{s}}\left(\frac{\partial V}{\partial x}+\frac{\partial A_{x}}{\partial t}\right)\right]-\frac{1}{\beta c} \frac{d \mathcal{E}}{d l} x^{\prime}+P G \xi_{x}, \\
P_{s}^{\prime} y^{\prime}+P_{s} y^{\prime \prime} & =-e\left[\frac{\partial A_{y}}{\partial s}-\frac{\partial A_{s}}{\partial y}+x^{\prime}\left(\frac{\partial A_{y}}{\partial x}-\frac{\partial A_{x}}{\partial y}\right)+\frac{m \gamma}{P_{s}}\left(\frac{\partial V}{\partial y}+\frac{\partial A_{y}}{\partial t}\right)\right]-\frac{1}{\beta c} \frac{d \mathcal{E}}{d l} y^{\prime}+P G \xi_{y}, \\
P_{s}^{\prime} & =-e\left[x^{\prime}\left(\frac{\partial A_{s}}{\partial x}-\frac{\partial A_{x}}{\partial s}\right)+y^{\prime}\left(\frac{\partial A_{s}}{\partial y}-\frac{\partial A_{y}}{\partial s}\right)+\frac{m \gamma}{P_{s}}\left(\frac{\partial V}{\partial s}+\frac{\partial A_{s}}{\partial t}\right)\right]-\frac{1}{\beta c} \frac{d \mathcal{E}}{d l} .
\end{aligned}
$$

\section{APPROXIMATIONS TO THE EQUATIONS OF MOTION FOR AXISYMMETRIC FIELDS}

We now specialize to the case of axially symmetric static magnetic and rf electromagnetic fields. For a static magnetic field, the vector potential in the region of the beam must satisfy the equation

$$
\nabla \times\left(\nabla \times \mathbf{A}_{M}\right)=0 .
$$

The solution to this equation, in cylindrical coordinates, for an axisymmetric magnetic field of solenoidal form, is

$$
\begin{aligned}
A_{M, \phi}(r, s) & =\frac{1}{2} \sum_{n=1,3,5, \ldots}^{\infty} a_{n} r^{n} B^{(n-1)}(s) \\
& =\frac{r}{2} B(s)-\frac{r^{3}}{16} B^{\prime \prime}(s)+\cdots,
\end{aligned}
$$

in which

$$
a_{1}=1, \quad a_{n}=-\frac{a_{n-2}}{n^{2}-1} .
$$

This potential gives the following static field, in Cartesian coordinates:

$$
\begin{aligned}
& B_{x}(x, y, s)=-\frac{x B^{\prime}(s)}{2}+\frac{x\left(x^{2}+y^{2}\right) B^{\prime \prime \prime}(s)}{16}+\cdots, \\
& B_{y}(x, y, s)=-\frac{y B^{\prime}(s)}{2}+\frac{y\left(x^{2}+y^{2}\right) B^{\prime \prime \prime}(s)}{16}+\cdots \\
& B_{s}(x, y, s)=B(s)-\frac{\left(x^{2}+y^{2}\right) B^{\prime \prime}(s)}{4}+\cdots
\end{aligned}
$$

We see that $B(s)$ is the on-axis solenoidal magnetic field.

For the rf electromagnetic field, we will work in a gauge for which the scalar potential $V=0$. Then, the vector potential must satisfy the equation

$$
\nabla \times\left(\nabla \times \mathbf{A}_{\mathrm{rf}}\right)+\frac{\omega^{2}}{c^{2}} \mathbf{A}_{\mathrm{rf}}=0,
$$

in which $\omega$ is the frequency of the rf field. The solution to this equation, in cylindrical coordinates, for the fundamental axisymmetric accelerating mode of a standing wave $\mathrm{rf}$ cavity, is

$$
\vec{A}_{\mathrm{rf}}(r, s, t)=\frac{1}{\omega} \vec{a}(r, s) \cos (\omega t+\phi)
$$

in which $\phi$ represents an arbitrary phase, the rf wave number is $\kappa=\omega / c$, and

$$
\begin{aligned}
& a_{s}(r, s)=E(s)+\sum_{n=1}^{\infty}(\kappa r)^{2 n} E_{2 n}(s), \\
& a_{r}(r, s)=\sum_{n=1}^{\infty}(\kappa r)^{2 n-1} E_{2 n-1}(s),
\end{aligned}
$$

with

$$
\begin{aligned}
& E_{0}(s)=E(s) \\
& E_{n}(s)=-\frac{E_{n-2}(s)+\frac{E_{n-2}^{\prime \prime}(s)}{\kappa^{2}}}{n^{2}} \text { for } n \text { even, } n>0 \\
& E_{n}(s)=-\frac{E_{n-1}^{\prime}(s)}{2 \kappa n} \quad \text { for } n \text { odd }
\end{aligned}
$$

The first few terms in the potential are

$$
\begin{aligned}
a_{s}(r, s)= & E(s)-\frac{r^{2}}{4}\left[E^{\prime \prime}(s)+\kappa^{2} E(s)\right] \\
& +\frac{r^{4}}{64}\left[E^{(4)}(s)+2 \kappa^{2} E^{\prime \prime}(s)+\kappa^{4} E(s)\right]+\cdots,
\end{aligned}
$$

$a_{r}(r, s)=-\frac{r}{2} E^{\prime}(s)+\frac{r^{3}}{16}\left[E^{(3)}(s)+\kappa^{2} E^{\prime}(s)\right]+\cdots$.

This potential gives the following rf fields, in Cartesian coordinates: 


$$
\begin{aligned}
& B_{x}(x, y, s, t)=-y \frac{\cos (\omega t+\phi)}{c}\left\{\frac{1}{2} E(s) \kappa+\frac{1}{16 \kappa}\left(x^{2}+y^{2}\right)\left[\kappa^{2} E^{\prime \prime}(s)+E^{(4)}(s)\right]\right\}+\cdots, \\
& B_{y}(x, y, s, t)=x \frac{\cos (\omega t+\phi)}{c}\left\{\frac{1}{2} E(s) \kappa+\frac{1}{16 \kappa}\left(x^{2}+y^{2}\right)\left[\kappa^{2} E^{\prime \prime}(s)+E^{(4)}(s)\right]\right\}+\cdots, \\
& B_{s}(x, y, s, t)=0 \\
& E_{x}(x, y, s, t)=x \sin (\omega t+\phi)\left\{-\frac{1}{2} E^{\prime}(s)+\frac{1}{16}\left(x^{2}+y^{2}\right)\left[\kappa^{2} E^{\prime}(s)+E^{(3)}(s)\right]\right\}+\cdots, \\
& E_{y}(x, y, s, t)=y \sin (\omega t+\phi)\left\{-\frac{1}{2} E^{\prime}(s)+\frac{1}{16}\left(x^{2}+y^{2}\right) \kappa\left[\kappa^{2} E^{\prime}(s)+E^{(3)}(s)\right]\right\}+\cdots, \\
& E_{s}(x, y, s, t)=\sin (\omega t+\phi)\left\{E(s)-\frac{1}{4}\left(x^{2}+y^{2}\right)\left[\kappa^{2} E(s)+E^{\prime \prime}(s)\right]\right\}+\cdots
\end{aligned}
$$

We see that $E(s)$ is the peak on-axis longitudinal accelerating field.

We now make the first approximation, which is to keep only the terms in the field equations, Eqs. (4.4) and (4.10), that are linear in the transverse coordinates. Since we will not be concerned with the details of the longitudinal dynamics here, we consider only the synchronous particle, and make the replacement $\omega t+\phi \rightarrow \phi_{s}$, where $\phi_{s}$ is the synchronous phase. The equations of motion, Eq. (3.13), then become

$$
\begin{aligned}
P_{s} x^{\prime \prime}= & \frac{e}{2}\left\{y B^{\prime}\left(1+x^{\prime 2}\right)+y^{\prime}\left(2 B-x x^{\prime} B^{\prime}\right)-\frac{m \gamma}{P_{s}} \sin \phi_{s}\left(2 E x^{\prime}+x E^{\prime}\right)-\left(\frac{\kappa E \cos \phi_{s}}{c}\right)\left[x\left(1+x^{\prime 2}\right)+y x^{\prime} y^{\prime}\right]\right\} \\
& +P G \xi_{x}, \\
P_{s} y^{\prime \prime}= & -\frac{e}{2}\left\{x B^{\prime}\left(1+y^{\prime 2}\right)+x^{\prime}\left(2 B-y y^{\prime} B^{\prime}\right)+\frac{m \gamma}{P_{s}} \sin \phi_{s}\left(2 E y^{\prime}+y E^{\prime}\right)+\left(\frac{\kappa E \cos \phi_{s}}{c}\right)\left[y\left(1+y^{\prime 2}\right)+x x^{\prime} y^{\prime}\right]\right\} \\
& +P G \xi_{y}, \\
P_{s}^{\prime}= & e E \sin \phi_{s} \frac{m \gamma}{P_{s}}+\left(\frac{\kappa e E \cos \phi_{s}}{2 c}\right)\left(x^{\prime} x+y^{\prime} y\right)-\frac{1}{\beta c} \frac{d \mathcal{E}}{d l}+\frac{e B^{\prime}\left(x y^{\prime}-y x^{\prime}\right)}{2} .
\end{aligned}
$$

The second approximation is the paraxial approximation: we linearize the equations of motion in the transverse angles $x^{\prime}$ and $y^{\prime}$. Then, $P / l^{\prime}=P_{s} \approx P$, and the equations of motion become

$$
\begin{aligned}
& x^{\prime \prime}=\frac{e}{2 P}\left[y B^{\prime}+2 y^{\prime} B-\frac{m \gamma \sin \phi_{s}\left(2 E x^{\prime}+x E^{\prime}\right)}{P}-x\left(\frac{\kappa E \cos \phi_{s}}{c}\right)\right]+G \xi_{x}, \\
& y^{\prime \prime}=-\frac{e}{2 P}\left[x B^{\prime}+2 x^{\prime} B+\frac{m \gamma \sin \phi_{s}\left(2 E y^{\prime}+y E^{\prime}\right)}{P}+y\left(\frac{\kappa E \cos \phi_{s}}{c}\right)\right]+G \xi_{y}, \\
& P^{\prime}=\frac{1}{\beta c}\left(e E \sin \phi_{s}-\frac{d \mathcal{E}}{d s}\right)+\left(\frac{\kappa e E \cos \phi_{s}}{2 c}\right)\left(x^{\prime} x+y^{\prime} y\right)+\frac{e B^{\prime}\left(x y^{\prime}-y x^{\prime}\right)}{2} .
\end{aligned}
$$

In this approximation, the $s$ component of the angular momentum is

$$
L=P\left(x y^{\prime}-y x^{\prime}\right)+\frac{e B}{2}\left(x^{2}+y^{2}\right)
$$

We now make a third approximation: we neglect the (nonlinear) last terms in the last line in Eq. (4.12). The momentum is then purely a function of $s$, satisfying the equation

$$
\frac{d P}{d s}=\frac{1}{\beta c}\left(e E \sin \phi_{s}-\frac{d \mathcal{E}}{d s}\right) .
$$

In a typical ionization cooling channel, the energy loss takes place in discrete absorbers, which are located between the rf cavities that restore the longitudinal momentum. According to Eq. (4.14), there is then a modulation of the momentum along the channel. However, this modulation is typically no more than a few percent, and will be ignored here. We thus take $P$ to be constant, and equal to the input momentum. Introducing the variables

$$
k(s)=\frac{e B(s)}{2 P}, \quad \eta(s)=\frac{e E(s) \sin \phi_{s}}{P \beta c},
$$

the first two lines in Eq. (4.12), which describe the transverse dynamics, become 


$$
\begin{aligned}
& x^{\prime \prime}=-\frac{1}{2}\left(\eta^{\prime}+\Pi \eta\right) x+y k^{\prime}+2 k y^{\prime}+G \xi_{x}-\eta x^{\prime}, \\
& y^{\prime \prime}=-\frac{1}{2}\left(\eta^{\prime}+\Pi \eta\right) y-x k^{\prime}-2 k x^{\prime}+G \xi_{y}-\eta y^{\prime},
\end{aligned}
$$

in which

$$
\Pi=\kappa \beta \cot \phi_{s}=\frac{2 \pi \beta \cot \phi_{s}}{\lambda_{\mathrm{rf}}},
$$

with $\lambda_{\text {rf }}$ being the wavelength of the rf field.

In developing the solution to these equations, it is convenient to transform to a rotating coordinate system. This is the conventional approach used to analyze particle motion in a solenoidal field. This coordinate transformation involves defining a new set of coordinates, which are rotated (clockwise) relative to $\{x, y\}$ by the angle $\theta_{r}(s)=$ $\int_{0}^{s} k(s) d s$. The rotated coordinates are

$$
\begin{aligned}
& x_{r}=x \cos \theta_{r}-y \sin \theta_{r}, \\
& y_{r}=y \cos \theta_{r}+x \sin \theta_{r} .
\end{aligned}
$$

The result of transforming Eq. (4.16) to the rotating coordinate system is

$$
\begin{aligned}
x_{r}^{\prime \prime}=- & {\left[k^{2}+\frac{1}{2}\left(\eta^{\prime}+\Pi \eta\right)\right] x_{r}-\eta k y_{r}-\eta x_{r}^{\prime} } \\
& +G\left(\cos \theta_{r} \xi_{x}-\sin \theta_{r} \xi_{y}\right), \\
y_{r}^{\prime \prime}=- & {\left[k^{2}+\frac{1}{2}\left(\eta^{\prime}+\Pi \eta\right)\right] y_{r}+\eta k x_{r}-\eta y_{r}^{\prime} } \\
& +G\left(\cos \theta_{r} \xi_{y}+\sin \theta_{r} \xi_{x}\right) .
\end{aligned}
$$

In the rotating frame, the angular momentum is just $L_{r}=$ $P\left(x_{r} y_{r}^{\prime}-y_{r} x_{r}^{\prime}\right)$. In the following, we will always work in the rotating coordinate system, and we will drop the subscripts on $x, y$, and $L$.

\section{SOLUTION OF THE EQUATIONS OF MOTION}

The dynamical equations, Eq. (4.19), are stochastic differential equations. The appropriate way to describe the motion of an ensemble of particles, subject to these dynamical equations of motion, is in terms of the distribution function $W\left(x, x^{\prime}, y, y^{\prime}, s\right)$. This distribution function represents the phase space density of an ensemble of particles, corresponding to the ensemble of realizations of the stochastic noise. In order to develop this distribution function, we must first find solutions to the dynamical equations given in Eq. (4.19), with the stochastic terms set to zero. To do so, we introduce the complex variable

$$
u(s)=[x(s)+i y(s)] \exp \left(\frac{\tilde{n}(s)}{2}\right)
$$

in which

$$
\tilde{n}(s)=\int_{0}^{s} \eta\left(s^{\prime}\right) d s^{\prime}
$$

Substituting this into Eq. (4.19), with the stochastic terms set to zero, gives the following differential equation for $u$ :

$$
u^{\prime \prime}=-\frac{1}{4}\left(\eta^{2}+4 i \eta k-2 \eta \Pi-4 k^{2}\right) u .
$$

Following the Courant-Snyder [6] procedure for the solution of this complex Hill's equation, the two linearly independent solutions for $u$ are

$$
u_{ \pm}(s)=\sqrt{\beta_{ \pm}(s)} \exp \left[ \pm i \Phi_{ \pm}(s) \pm \frac{1}{2} \int_{0}^{s} d s^{\prime} \eta\left(s^{\prime}\right) k\left(s^{\prime}\right) \beta_{ \pm}\left(s^{\prime}\right)\right]
$$

in which

$$
\Phi_{ \pm}(s)=\int_{0}^{s} \frac{d s^{\prime}}{\beta_{ \pm}\left(s^{\prime}\right)} .
$$

The pair of lattice functions $\beta_{ \pm}(s)$ are solutions to the following differential equations:

$$
\beta_{ \pm}^{\prime \prime}=\frac{1}{2 \beta_{ \pm}}\left\{\left(4+\beta_{ \pm}^{2} \eta^{2}\right)\left(1-\beta_{ \pm}^{2} k^{2}\right)+\beta_{ \pm}^{\prime}\left(\beta_{ \pm}^{\prime} \mp 2 \beta_{ \pm}^{2} \eta k\right)-2 \beta_{ \pm}^{2} \eta \Pi \mp 2 \beta_{ \pm}^{2}\left[\beta_{ \pm} \eta k\right]^{\prime}\right\} .
$$

Note that Eq. (5.6) involves only the electric and magnetic fields. The lattice functions are completely specified by obtaining solutions of Eq. (5.6) that satisfy given initial conditions on $\beta_{ \pm}(0)$ and $\beta_{ \pm}^{\prime}(0)$ (for a periodic channel, these will be periodic boundary conditions; see Sec. XI). For $\eta=0$, Eq. (5.6) reduces to the usual differential equation for the lattice function $\beta$.

The derivatives of Eq. (5.4) can be written as

$$
u_{ \pm}^{\prime}=\left( \pm i-\alpha_{ \pm}+\frac{\beta_{ \pm} \eta}{2}\right) \frac{u_{ \pm}}{\beta_{ \pm}}
$$

in which

$$
\alpha_{ \pm}=\frac{-\beta_{ \pm}^{\prime}+\beta_{ \pm} \eta\left(1 \mp \beta_{ \pm} k\right)}{2} .
$$

The differential equations [(5.6)] may be written as an equation for $\alpha_{ \pm}$:

$$
\begin{aligned}
\alpha_{ \pm}^{\prime}= & -\gamma_{ \pm}+\beta_{ \pm} k^{2} \mp \alpha_{ \pm} \beta_{ \pm} \eta k \\
& +\frac{1}{2} \beta_{ \pm} \eta^{\prime}+\frac{1}{2} \Pi \beta_{ \pm} \eta
\end{aligned}
$$


in which

$$
\gamma_{ \pm}=\frac{1+\alpha_{ \pm}^{2}}{\beta_{ \pm}}
$$

The Wronskian of the differential equation, Eq. (5.3), is the constant

$$
C=u_{+} u_{-}^{\prime}-u_{-} u_{+}^{\prime}=\frac{-\left(i+\alpha_{-}\right) \beta_{+}+\left(-i+\alpha_{+}\right) \beta_{-}}{\sqrt{\beta_{+} \beta_{-}}} \exp \left[i\left(\Phi_{+}-\Phi_{-}\right)+\zeta_{0}-\tilde{n}\right],
$$

in which

$$
\begin{aligned}
& \zeta_{0}(s)=\frac{\zeta_{+}(s)+\zeta_{-}(s)}{2} \\
& \zeta_{ \pm}(s)=\int_{0}^{s} d s^{\prime} \eta\left(s^{\prime}\right)\left[1 \pm k\left(s^{\prime}\right) \beta_{ \pm}\left(s^{\prime}\right)\right]=\tilde{n}(s) \pm \int_{0}^{s} d s^{\prime} \eta\left(s^{\prime}\right) k\left(s^{\prime}\right) \beta_{ \pm}\left(s^{\prime}\right) .
\end{aligned}
$$

The squared modulus of this constant is

$$
|C|^{2}=\delta(s) \exp \left(2 \zeta_{0}-2 \tilde{n}\right)
$$

in which

$$
\delta(s)=\left\{2\left[1-\alpha_{+}(s) \alpha_{-}(s)\right]+\beta_{+}(s) \gamma_{-}(s)+\beta_{-}(s) \gamma_{+}(s)\right\} .
$$

From Eq. (5.13), we have $|C|^{2}=\delta(0)$. The general solution to Eq. (5.3) may be written as

$$
u=(a+i b) u_{+}+(c+i d) u_{-},
$$

in which $a, b, c$, and $d$ are real constants, and $u_{ \pm}$is given by Eq. (5.4). Using Eq. (5.15), Eq. (5.1) may be written as

$$
\begin{aligned}
x+i y & =u \exp \left(-\frac{\tilde{n}}{2}\right)=\exp \left(-\frac{\tilde{n}}{2}\right)\left[(a+i b) u_{+}+(c+i d) u_{-}\right] \\
& =(a+i b) \sqrt{\beta_{+}} \exp \left(-\tilde{n}+\frac{\zeta_{+}}{2}+i \Phi_{+}\right)+(c+i d) \sqrt{\beta_{-}} \exp \left(-\tilde{n}+\frac{\zeta_{-}}{2}-i \Phi_{-}\right) .
\end{aligned}
$$

The solutions to Eq. (4.19), with the stochastic terms set to zero, in terms of real quantities, are then

$$
\begin{aligned}
& x=\exp (-\tilde{n})\left[\sqrt{\beta_{+}} \exp \left(\frac{\zeta_{+}}{2}\right)\left(a \cos \Phi_{+}-b \sin \Phi_{+}\right)+\sqrt{\beta_{-}} \exp \left(\frac{\zeta_{-}}{2}\right)\left(c \cos \Phi_{-}+d \sin \Phi_{-}\right)\right], \\
& y=\exp (-\tilde{n})\left[\sqrt{\beta_{+}} \exp \left(\frac{\zeta_{+}}{2}\right)\left(b \cos \Phi_{+}+a \sin \Phi_{+}\right)+\sqrt{\beta_{-}} \exp \left(\frac{\zeta_{-}}{2}\right)\left(d \cos \Phi_{-}-c \sin \Phi_{-}\right)\right],
\end{aligned}
$$

in which the constants $a, b, c$, and $d$ are determined by the initial conditions. The relationship between $\mathbf{x}=\left\{x, x^{\prime}, y, y^{\prime}\right\}=\left\{x_{1}, x_{2}, x_{3}, x_{4}\right\}$ and the initial conditions $\mathbf{x}_{0}=\left\{x_{0}, x_{0}^{\prime}, y_{0}, y_{0}^{\prime}\right\}=\left\{x_{01}, x_{02}, x_{03}, x_{04}\right\}$ can be written as the matrix equation

$$
\mathbf{x}(s)=\mathbf{T}(s, 0) \mathbf{x}_{0},
$$

in which $\mathbf{T}(s, 0)$ is the four-dimensional transport matrix from $s=0$ to $s$, whose elements can be derived from Eq. (5.17).

\section{SOLUTION OF THE FOKKER-PLANCK EQUATION}

The distribution function mentioned above, $W\left(x, x^{\prime}, y, y^{\prime}, s\right)$, is the solution to the Fokker-Planck equation. For the set of dynamical variables $\left\{x_{i}\right\}$, which obey stochastic differential equations of the generalized Langevin form,

$$
x_{i}^{\prime}=K_{i}(\mathbf{x}, s)+\sum_{j} Q_{i j}(\mathbf{x}, s) \xi_{j}(s),
$$

the Fokker-Planck equation is [10]

$$
\begin{aligned}
\frac{\partial W}{\partial s}= & -\sum_{i} \frac{\partial}{\partial x_{i}}\left(\left[K_{i}+\frac{1}{2} \sum_{j} \sum_{k} \frac{\partial Q_{i k}}{\partial x_{j}} Q_{k j}\right] W\right) \\
& +\frac{1}{2} \sum_{i} \sum_{j} \frac{\partial}{\partial x_{i}} \frac{\partial}{\partial x_{j}} \sum_{k}\left[Q_{i k} Q_{k j} W\right] .
\end{aligned}
$$

In the absence of damping and stochastic effects, this reduces to the Vlasov equation. For this application, writing Eq. (4.19) in the general form of Eq. (6.1), we have 


$$
\mathbf{x}^{\prime}=\left(\begin{array}{c}
x^{\prime} \\
x^{\prime \prime} \\
y^{\prime} \\
y^{\prime \prime}
\end{array}\right)=\left(\begin{array}{c}
x^{\prime} \\
-\left[k^{2}+\frac{1}{2}\left(\eta^{\prime}+\Pi \eta\right)\right] x-\eta k y-\eta x^{\prime}+G\left(\cos \theta_{r} \xi_{x}-\sin \theta_{r} \xi_{y}\right) \\
-\left[k^{2}+\frac{1}{2}\left(\eta^{\prime}+\Pi \eta\right)\right] y+\eta k x-\eta y^{\prime}+G\left(\cos \theta_{r} \xi_{y}+\sin \theta_{r} \xi_{x}\right)
\end{array}\right),
$$

so we can identify

$$
\mathbf{K}=\left(\begin{array}{c}
x_{2} \\
-\left[k^{2}+\frac{1}{2}\left(\eta^{\prime}+\Pi \eta\right)\right] x_{1}-\eta k x_{3}-\eta x_{2} \\
x_{4} \\
-\left[k^{2}+\frac{1}{2}\left(\eta^{\prime}+\Pi \eta\right)\right] x_{3}+\eta k x_{1}-\eta x_{4}
\end{array}\right), \quad \mathbf{Q}=G\left(\begin{array}{cc}
0 & 0 \\
\cos \theta_{r} & -\sin \theta_{r} \\
0 & 0 \\
\sin \theta_{r} & \cos \theta_{r}
\end{array}\right)
$$

The Fokker-Planck equation, for this case in which $\mathbf{Q}$ is independent of $\mathbf{x}$, is

$$
\begin{aligned}
\frac{\partial W}{\partial s}= & -W \sum_{i} \frac{\partial K_{i}}{\partial x_{i}}-\sum_{i} K_{i} \frac{\partial W}{\partial x_{i}}+\frac{1}{2} \sum_{i} \sum_{j} \sum_{k}\left[Q_{i k} Q_{k j}\right] \frac{\partial}{\partial x_{i}} \frac{\partial}{\partial x_{j}} W \\
= & 2 W \eta-x^{\prime} \frac{\partial W}{\partial x}-y^{\prime} \frac{\partial W}{\partial y}+\left\{\left[k^{2}+\frac{1}{2}\left(\eta^{\prime}+\Pi \eta\right)\right] x+\eta k y+\eta x^{\prime}\right\} \frac{\partial W}{\partial x^{\prime}} \\
& +\left\{\left[k^{2}+\frac{1}{2}\left(\eta^{\prime}+\Pi \eta\right)\right] y-\eta k x+\eta y^{\prime}\right\} \frac{\partial W}{\partial y^{\prime}}+\frac{G^{2}}{2}\left(\frac{\partial^{2} W}{\partial x^{\prime 2}}+\frac{\partial^{2} W}{\partial y^{\prime 2}}\right) .
\end{aligned}
$$

We will proceed to solve this equation for the phase space density $W\left(x, x^{\prime}, y, y^{\prime}, s\right)$. The method to be used is one originally developed for an analysis of Brownian motion [7]. We transform from the normal phase space variables $\mathbf{x}=\left\{x, x^{\prime}, y, y^{\prime}\right\}$ to a new set $\boldsymbol{\chi}=\left\{\chi_{1}, \chi_{2}, \chi_{3}, \chi_{4}\right\}$. The following equations and their first derivatives define this transformation:

$$
\begin{aligned}
& x=\sqrt{|C|} \exp (-\tilde{n})\left[\sqrt{\beta_{+}} \exp \left(\frac{\zeta_{+}}{2}\right)\left(\chi_{1} \cos \Phi_{+}-\chi_{2} \sin \Phi_{+}\right)+\sqrt{\beta_{-}} \exp \left(\frac{\zeta_{-}}{2}\right)\left(\chi_{3} \cos \Phi_{-}+\chi_{4} \sin \Phi_{-}\right)\right], \\
& y=\sqrt{|C|} \exp (-\tilde{n})\left[\sqrt{\beta_{+}} \exp \left(\frac{\zeta_{+}}{2}\right)\left(\chi_{2} \cos \Phi_{+}+\chi_{1} \sin \Phi_{+}\right)+\sqrt{\beta_{-}} \exp \left(\frac{\zeta_{-}}{2}\right)\left(\chi_{4} \cos \Phi_{-}-\chi_{3} \sin \Phi_{-}\right)\right] .
\end{aligned}
$$

Comparison with Eq. (5.17) shows that the $\mathcal{\chi}$ quantities are just proportional to the constants $a, b$, $c$, and $d$, and so will be constants of the motion in the absence of any stochastic processes. The transformation of variables given in Eq. (6.6) can be written as a matrix equation

$$
\boldsymbol{\chi}=\mathbf{M}(s) \mathbf{x}(s)
$$

in which the elements of $\mathbf{M}^{-1}(s)$ can be obtained from inspection of Eq. (6.6) and its first derivatives.

We now transform the Fokker-Planck equation, Eq. (6.5), into these new variables, obtaining, after considerable algebra,

$$
\begin{aligned}
\frac{\partial W}{\partial s}-2 \eta W= & \frac{G^{2}}{2|C|} \beta_{-} \exp \left(\zeta_{-}\right)\left(\frac{\partial^{2} W}{\partial \chi_{1}^{2}}+\frac{\partial^{2} W}{\partial \chi_{2}^{2}}\right)+\frac{G^{2}}{2|C|} \beta_{+} \exp \left(\zeta_{+}\right)\left(\frac{\partial^{2} W}{\partial \chi_{3}^{2}}+\frac{\partial^{2} W}{\partial \chi_{4}^{2}}\right) \\
& -\left(\frac{\partial^{2} W}{\partial \chi_{1} \partial \chi_{3}}+\frac{\partial^{2} W}{\partial \chi_{2} \partial \chi_{4}}\right)\left[\frac{G^{2}}{|C|} \sqrt{\beta_{+} \beta_{-}} \cos \left(2 \Phi_{0}\right) \exp \left(\zeta_{0}\right)\right] \\
& -\left(\frac{\partial^{2} W}{\partial \chi_{1} \partial \chi_{4}}-\frac{\partial^{2} W}{\partial \chi_{2} \partial \chi_{3}}\right)\left[\frac{G^{2}}{|C|} \sqrt{\beta_{+} \beta_{-}} \sin \left(2 \Phi_{0}\right) \exp \left(\zeta_{0}\right)\right]
\end{aligned}
$$

in which

$$
\Phi_{0}=\frac{\Phi_{+}+\Phi_{-}}{2}
$$

Let $\omega$ be the distribution function in $\chi$. Then we have

$$
W=\omega|\operatorname{Det}[\mathbf{M}]|=\omega \exp (2 \tilde{n}) \Rightarrow \frac{\partial W}{\partial s}=\frac{\partial \omega}{\partial s} \exp (2 \tilde{n})+2 \eta W .
$$

In terms of $\omega$, Eq. (6.8) becomes 


$$
\begin{aligned}
\frac{\partial \omega}{\partial s}= & \frac{G^{2}}{2|C|} \beta_{-} \exp \left(\zeta_{-}\right)\left(\frac{\partial^{2} \omega}{\partial \chi_{1}^{2}}+\frac{\partial^{2} \omega}{\partial \chi_{2}^{2}}\right)+\frac{G^{2}}{2|C|} \beta_{+} \exp \left(\zeta_{+}\right)\left(\frac{\partial^{2} \omega}{\partial \chi_{3}^{2}}+\frac{\partial^{2} \omega}{\partial \chi_{4}^{2}}\right) \\
& -\left(\frac{\partial^{2} \omega}{\partial \chi_{1} \partial \chi_{3}}+\frac{\partial^{2} \omega}{\partial \chi_{2} \partial \chi_{4}}\right)\left[\frac{G^{2}}{|C|} \sqrt{\beta_{+} \beta_{-}} \cos \left(2 \Phi_{0}\right) \exp \left(\zeta_{0}\right)\right] \\
& -\left(\frac{\partial^{2} \omega}{\partial \chi_{1} \partial \chi_{4}}-\frac{\partial^{2} \omega}{\partial \chi_{2} \partial \chi_{3}}\right)\left[\frac{G^{2}}{|C|} \sqrt{\beta_{+} \beta_{-}} \sin \left(2 \Phi_{0}\right) \exp \left(\zeta_{0}\right)\right]
\end{aligned}
$$

This equation has the general form:

$$
\begin{aligned}
\frac{\partial \omega}{\partial s}= & \left(\frac{\partial^{2} \omega}{\partial \chi_{1}^{2}}+\frac{\partial^{2} \omega}{\partial \chi_{2}^{2}}\right) \phi_{1}(s)+\left(\frac{\partial^{2} \omega}{\partial \chi_{3}^{2}}+\frac{\partial^{2} \omega}{\partial \chi_{4}^{2}}\right) \phi_{2}(s)+\left(\frac{\partial^{2} \omega}{\partial \chi_{1} \partial \chi_{3}}+\frac{\partial^{2} \omega}{\partial \chi_{2} \partial \chi_{4}}\right) \phi_{3}(s) \\
& +\left(\frac{\partial^{2} \omega}{\partial \chi_{1} \partial \chi_{4}}-\frac{\partial^{2} \omega}{\partial \chi_{2} \partial \chi_{3}}\right) \phi_{4}(s) .
\end{aligned}
$$

We seek a solution to this equation corresponding to an initial delta-function distribution in $\mathcal{\chi}$, centered at

$$
\boldsymbol{\chi}_{0}=\mathbf{M}(0) \mathbf{x}_{0} .
$$

The normalized general solution to Eq. (6.12), with the initial condition that

$$
\lim _{s \rightarrow 0} \omega\left(\boldsymbol{\chi}, \chi_{0}, s\right)=\prod_{i} \delta\left(\chi_{i}-\chi_{0 i}\right),
$$

is

$$
\omega\left(\boldsymbol{\chi}, \boldsymbol{\chi}_{0}, s\right)=\frac{1}{(2 \pi)^{2} \Delta(s)} \exp \left[-\frac{1}{2 \Delta(s)} \sum_{i} \sum_{j}\left(\chi_{i}-\chi_{0 i}\right) A_{i j}(s)\left(\chi_{j}-\chi_{0 j}\right)\right]
$$

In this equation,

$$
\mathbf{A}(s)=\left(\begin{array}{cccc}
a(s) & 0 & c(s) & d(s) \\
0 & a(s) & -d(s) & c(s) \\
c(s) & -d(s) & b(s) & 0 \\
d(s) & c(s) & 0 & b(s)
\end{array}\right)
$$

The matrix $\mathbf{A}$ has a determinant

$$
\operatorname{Det}[\mathbf{A}]=\Delta(s)^{2}
$$

with

$$
\Delta(s)=a(s) b(s)-c(s)^{2}-d(s)^{2} .
$$

The matrix elements are given in terms of the functions in Eq. (6.12) by

$$
a(s)=2 \int_{0}^{s} \phi_{2}\left(s^{\prime}\right) d s^{\prime}, \quad b(s)=2 \int_{0}^{s} \phi_{1}\left(s^{\prime}\right) d s^{\prime}, \quad c(s)=-\int_{0}^{s} \phi_{3}\left(s^{\prime}\right) d s^{\prime}, \quad d(s)=-\int_{0}^{s} \phi_{4}\left(s^{\prime}\right) d s^{\prime} .
$$

Applying this to Eq. (6.11), we find for the matrix elements of $\mathbf{A}$ :

$$
\begin{aligned}
& a(s)=\frac{1}{|C|} \int_{0}^{s} G\left(s^{\prime}\right)^{2} \beta_{+}\left(s^{\prime}\right) \exp \left[\zeta_{+}\left(s^{\prime}\right)\right] d s^{\prime} \\
& b(s)=\frac{1}{|C|} \int_{0}^{s} G\left(s^{\prime}\right)^{2} \beta_{-}\left(s^{\prime}\right) \exp \left[\zeta_{-}\left(s^{\prime}\right)\right] d s^{\prime} \\
& c(s)=\frac{1}{|C|} \int_{0}^{s} G\left(s^{\prime}\right)^{2} \sqrt{\beta_{+}\left(s^{\prime}\right) \beta_{-}\left(s^{\prime}\right)} \exp \left[\zeta_{0}\left(s^{\prime}\right)\right] \cos \left[2 \Phi_{0}\left(s^{\prime}\right)\right] d s^{\prime}, \\
& d(s)=\frac{1}{|C|} \int_{0}^{s} G\left(s^{\prime}\right)^{2} \sqrt{\beta_{+}\left(s^{\prime}\right) \beta_{-}\left(s^{\prime}\right)} \exp \left[\zeta_{0}\left(s^{\prime}\right)\right] \sin \left[2 \Phi_{0}\left(s^{\prime}\right)\right] d s^{\prime}
\end{aligned}
$$




\section{SECOND MOMENTS OF THE DISTRIBUTION}

The matrix of second moments of the variables $\boldsymbol{\chi}$ can be written in terms of the matrix $\mathbf{A}$, using the following theorem [11].

A system of $n$ variables, having a normalized distribution function of the form

$$
f\left(x_{1}, x_{2}, x_{3} \ldots, x_{n}\right)=\frac{1}{(2 \pi)^{n / 2} \sqrt{|\operatorname{Det}[\mathbf{k}]|}} \exp \left[-\frac{1}{2} \sum_{i=1}^{n} \sum_{j=1}^{n} k_{i j}^{-1}\left(x_{i}-\bar{x}_{i}\right)\left(x_{j}-\bar{x}_{j}\right)\right]
$$

has a covariance matrix given by $k_{i j}=\overline{\left(x_{i}-\bar{x}_{i}\right)\left(x_{j}-\bar{x}_{j}\right)}=\overline{x_{i} x_{j}}-\bar{x}_{i} \bar{x}_{j}$. Thus, in our case, comparing Eq. (7.1) with Eq. (6.15), we see that the inverse of the covariance matrix for the variables $\chi_{i}$ is

$$
k_{i j}^{-1}=\frac{A_{i j}}{\Delta}
$$

and the covariance matrix $k_{i j}=\overline{\chi_{i} \chi_{j}}-\bar{\chi}_{i} \bar{\chi}_{j}=\overline{\chi_{i} \chi_{j}}-\chi_{0 i} \chi_{0 j}$ is

$$
\mathbf{k}=\left(\begin{array}{cccc}
b & 0 & -c & -d \\
0 & b & d & -c \\
-c & d & a & 0 \\
-d & -c & 0 & a
\end{array}\right)
$$

with $\operatorname{Det}[\mathbf{k}]=\Delta^{2}$.

From Eq. (7.3), we can see that the moments of certain combinations of the variables $\chi_{i}$ have simple forms:

$$
\begin{aligned}
& \overline{\chi_{1}^{2}}+\overline{\chi_{2}^{2}}-\left(\chi_{10}^{2}+\chi_{20}^{2}\right)=2 b=\frac{2}{|C|} \int_{0}^{s} G\left(s^{\prime}\right)^{2} \beta_{-}\left(s^{\prime}\right) \exp \left[\zeta_{-}\left(s^{\prime}\right)\right] d s^{\prime}, \\
& \overline{\chi_{3}^{2}}+\overline{\chi_{4}^{2}}-\left(\chi_{30}^{2}+\chi_{40}^{2}\right)=2 a=\frac{2}{|C|} \int_{0}^{s} G\left(s^{\prime}\right)^{2} \beta_{+}\left(s^{\prime}\right) \exp \left[\zeta_{+}\left(s^{\prime}\right)\right] d s^{\prime} .
\end{aligned}
$$

We can relate these combinations to the normal phase space variables using Eq. (6.6):

$$
\begin{aligned}
& \chi_{1}^{2}+\chi_{2}^{2}=\frac{1}{|C|} \exp \left(\zeta_{-}\right)\left[\left(x^{\prime 2}+y^{\prime 2}\right) \beta_{-}+\left(x^{2}+y^{2}\right) \gamma_{-}+2 \alpha_{-}\left(x x^{\prime}+y y^{\prime}\right)+2 \frac{L}{P}\right], \\
& \chi_{3}^{2}+\chi_{4}^{2}=\frac{1}{|C|} \exp \left(\zeta_{+}\right)\left[\left(x^{\prime 2}+y^{\prime 2}\right) \beta_{+}+\left(x^{2}+y^{2}\right) \gamma_{+}+2 \alpha_{+}\left(x x^{\prime}+y y^{\prime}\right)-2 \frac{L}{P}\right] .
\end{aligned}
$$

Let us define

$$
J_{ \pm}=\frac{\left(x^{\prime 2}+y^{\prime 2}\right) \beta_{ \pm}+\left(x^{2}+y^{2}\right) \gamma_{ \pm}+2 \alpha_{ \pm}\left(x x^{\prime}+y y^{\prime}\right)}{2} \mp \frac{L}{P} .
$$

These quantities bear a certain resemblance to Courant-Snyder invariants. They are constants of the motion in the absence of the absorbing and scattering medium, and the electric field. We will call them "pseudoinvariants." From Eq. (7.5), we have

$$
\overline{\chi_{1}^{2}}+\overline{\chi_{2}^{2}}=\frac{2}{|C|} \exp \left(\zeta_{-}\right) \overline{J_{-}}, \quad \overline{\chi_{3}^{2}}+\overline{\chi_{4}^{2}}=\frac{2}{|C|} \exp \left(\zeta_{+}\right) \overline{\boldsymbol{J}_{+}} .
$$

From Eq. (7.4), the averages of the pseudoinvariants are

$$
\overline{J_{ \pm}(s)}=I_{ \pm}(s)+J_{ \pm}(s),
$$

in which

$$
J_{ \pm}(s)=\exp \left(-\zeta_{ \pm}\right) \int_{0}^{s} G\left(s^{\prime}\right)^{2} \beta_{ \pm}\left(s^{\prime}\right) \exp \left[\zeta_{ \pm}\left(s^{\prime}\right)\right] d s^{\prime},
$$

and in which

$$
\begin{aligned}
I_{ \pm}(s) & =I_{ \pm 0} \exp \left(-\zeta_{ \pm}\right) \\
I_{ \pm 0} & =\frac{\left(x_{0}^{\prime 2}+y_{0}^{\prime 2}\right) \beta_{ \pm}(0)+\left(x_{0}^{2}+y_{0}^{2}\right) \gamma_{ \pm}(0)+2 \alpha_{ \pm}(0)\left(x_{0} x_{0}^{\prime}+y_{0} y_{0}^{\prime}\right)}{2} \mp \frac{L_{0}}{P}
\end{aligned}
$$




\section{DISTRIBUTION FUNCTION AND SECOND MOMENTS FOR THE NORMAL PHASE SPACE VARIABLES}

We may also obtain the distribution function and the covariance matrix for the normal phase space variables. From Eqs. (6.7) and (6.13) we have

$$
\boldsymbol{\chi}-\boldsymbol{\chi}_{0}=\mathbf{M}(s) \mathbf{x}(s)-\mathbf{M}(0) \mathbf{x}_{0}=\mathbf{M}(s)\left[\mathbf{x}(s)-\mathbf{M}^{-1}(s) \mathbf{M}(0) \mathbf{x}_{0}\right] .
$$

Then the quadratic form appearing in Eq. (6.15) is

$$
\begin{aligned}
\left(\boldsymbol{\chi}-\boldsymbol{\chi}_{0}\right) \mathbf{A}(s)\left(\boldsymbol{\chi}-\boldsymbol{\chi}_{0}\right) & =\left[\mathbf{x}(s)-\mathbf{M}^{-1}(s) \mathbf{M}(0) \mathbf{x}_{0}\right] \mathbf{M}^{\mathrm{T}}(s) \mathbf{A}(s) \mathbf{M}(s)\left[\mathbf{x}(s)-\mathbf{M}^{-1}(s) \mathbf{M}(0) \mathbf{x}_{0}\right] \\
& =[\mathbf{x}(s)-\overline{\mathbf{x}}(s)] \mathbf{Q}(s)[\mathbf{x}(s)-\overline{\mathbf{x}}(s)],
\end{aligned}
$$

in which $\mathbf{M}^{\mathrm{T}}(s)$ is the transpose of $\mathbf{M}(s)$. The matrix $\mathbf{Q}(s)=\mathbf{M}^{\mathrm{T}}(s) \mathbf{A}(s) \mathbf{M}(s)$ will be related to the covariance matrix of $\mathbf{x}=\left\{x, x^{\prime}, y, y^{\prime}\right\}$. The first moments are given by

$$
\overline{\mathbf{x}}(s)=\mathbf{M}^{-1}(s) \mathbf{M}(0) \mathbf{x}_{0} .
$$

The first moments can be written more simply in terms of the transport matrix introduced in Eq. (5.18). From Eq. (6.7) we have

$$
\begin{aligned}
\mathbf{x}\left(s_{2}\right) & =\mathbf{M}^{-1}\left(s_{2}\right) \boldsymbol{\chi}=\mathbf{T}\left(s_{2}, s_{1}\right) \mathbf{x}\left(s_{1}\right)=\mathbf{T}\left(s_{2}, s_{1}\right) \mathbf{M}^{-1}\left(s_{1}\right) \boldsymbol{\chi} \\
& \Rightarrow \mathbf{M}^{-1}\left(s_{2}\right)=\mathbf{T}\left(s_{2}, s_{1}\right) \mathbf{M}^{-1}\left(s_{1}\right) \Rightarrow \mathbf{T}\left(s_{2}, s_{1}\right)=\mathbf{M}^{-1}\left(s_{2}\right) \mathbf{M}\left(s_{1}\right) .
\end{aligned}
$$

So Eq. (8.3) can also be written as

$$
\overline{\mathbf{x}}(s)=\mathbf{T}(s, 0) \mathbf{x}_{0} .
$$

The distribution function in $\mathbf{x}=\left\{x, x^{\prime}, y, y^{\prime}\right\}$ is given from Eqs. (6.10), (6.15), and (8.2) by

$$
W\left(\mathbf{x}, \mathbf{x}_{0}, s\right)=\frac{\exp (2 \tilde{n})}{(2 \pi)^{2} \Delta} \exp \left(-\frac{(\mathbf{x}-\overline{\mathbf{x}}) \mathbf{Q}(\mathbf{x}-\overline{\mathbf{x}})}{2 \Delta}\right)=\frac{\exp (2 \tilde{n})}{(2 \pi)^{2} \Delta} \exp \left[-\frac{1}{2} \sum_{i, j=1}^{n} K_{i j}^{-1}\left(x_{i}-\bar{x}_{i}\right)\left(x_{j}-\bar{x}_{j}\right)\right],
$$

in which

$$
\mathbf{K}^{-1}=\frac{\mathbf{Q}}{\Delta}=\frac{\mathbf{M}^{\mathrm{T}} \mathbf{A} \mathbf{M}}{\Delta}
$$

So the covariance matrix is

$$
\mathbf{K}=\mathbf{M}^{-1}\left(\frac{\mathbf{A}}{\Delta}\right)^{-1}\left(\mathbf{M}^{\mathrm{T}}\right)^{-1}=\mathbf{M}^{-1} \mathbf{k}\left(\mathbf{M}^{\mathrm{T}}\right)^{-1}
$$

with

$$
\operatorname{Det}[\mathbf{K}]=[\operatorname{Det}[\mathbf{M}]]^{-2} \operatorname{Det}[\mathbf{k}]=\Delta^{2} \exp (-4 \tilde{n})
$$

The elements of the covariance matrix $K_{i j}=\overline{x_{i} x_{j}}-\bar{x}_{i} \bar{x}_{j}$ then follow from Eqs. (8.8), (7.3), (6.7), and (6.6). They are

$$
\begin{aligned}
K_{x x} & =K_{y y}=\frac{1}{\delta}\left(\beta_{-} J_{+}+\beta_{+} J_{-}-2 \sqrt{\beta_{-} \beta_{+}} J_{c}\right), \\
K_{x x^{\prime}} & =K_{y y^{\prime}}=\frac{1}{\delta}\left[-\alpha_{-} J_{+}-\alpha_{+} J_{-}+\left(\alpha_{-} \sqrt{\frac{\beta_{+}}{\beta_{-}}}+\alpha_{+} \sqrt{\frac{\beta_{-}}{B_{+}}}\right) J_{c}+\left(\sqrt{\frac{\beta_{+}}{\beta_{-}}}+\sqrt{\frac{\beta_{-}}{\beta_{+}}}\right) J_{s}\right], \\
K_{x^{\prime} x^{\prime}} & =K_{y^{\prime} y^{\prime}}=\frac{1}{\delta}\left(\gamma_{-} J_{+}+\gamma_{+} J_{-}+2 \frac{\left(1-\alpha_{+} \alpha_{-}\right)}{\sqrt{\beta_{-} \beta_{+}}} J_{c}-\frac{2\left(\alpha_{+}+\alpha_{-}\right)}{\sqrt{\beta_{-} \beta_{+}}} J_{s}\right) \\
K_{x y} & =K_{x^{\prime} y^{\prime}}=0 \\
K_{x y^{\prime}} & =-K_{x^{\prime} y}=\frac{1}{\delta}\left[J_{-}-J_{+}-\left(\alpha_{-} \sqrt{\frac{\beta_{+}}{\beta_{-}}}-\alpha_{+} \sqrt{\frac{\beta_{-}}{\beta_{+}}}\right) J_{s}+\left(\sqrt{\frac{\beta_{+}}{\beta_{-}}}-\sqrt{\frac{\beta_{-}}{\beta_{+}}}\right) J_{c}\right] .
\end{aligned}
$$


In this equation, $J_{+}$and $J_{-}$are given by Eq. (7.9), and

$$
J_{c}(s)+i J_{s}(s)=\exp \left(-\zeta_{0}+2 i \Phi_{0}\right) \int_{0}^{s} G\left(s^{\prime}\right)^{2} \sqrt{\beta_{+}\left(s^{\prime}\right) \beta_{-}\left(s^{\prime}\right)} \exp \left[\zeta_{0}\left(s^{\prime}\right)-2 i \Phi_{0}\left(s^{\prime}\right)\right] d s^{\prime} .
$$

If we know $K_{i j}$, to find the second moments $\overline{x_{i} x_{j}}$, we need to know $\bar{x}_{i} \overline{x_{j}}$. Using Eq. (8.3), the matrix $\mathbf{X}$ of first moment products may be written as

$$
X_{i j}=\bar{x}_{i} \bar{x}_{j}=\sum_{l} \sum_{n} T_{i l}(s, 0) x_{0 l} T_{j n}(s, 0) x_{0 n}=\sum_{l} \sum_{n} T_{i l}(s, 0) x_{0 l} x_{0 n} T_{n j}^{T}(s, 0)
$$

More simply,

$$
\mathbf{X}(s)=\mathbf{T}(s, 0) \mathbf{X}_{0} \mathbf{T}^{\mathrm{T}}(s, 0),
$$

in which the initial value matrix is

$$
\mathbf{X}_{0}=\left(\begin{array}{cccc}
x_{0}^{2} & x_{0} x_{0}^{\prime} & x_{0} y_{0} & x_{0} y_{0}^{\prime} \\
x_{0}^{\prime} x_{0} & x_{0}^{\prime 2} & x_{0}^{\prime} y_{0} & x_{0}^{\prime} y_{0}^{\prime} \\
y_{0} x_{0} & y_{0} x_{0}^{\prime} & y_{0}^{2} & y_{0} y_{0}^{\prime} \\
y_{0}^{\prime} x_{0} & y_{0}^{\prime} x_{0}^{\prime} & y_{0}^{\prime} y_{0} & y_{0}^{\prime 2}
\end{array}\right)
$$

The equation

$$
\overline{x_{i} x_{j}}=K_{i j}+X_{i j}
$$

then specifies the second moments of a distribution, corresponding to an ensemble of particles, suffering multiple Coulomb scattering, but all with the same initial trajectory, given by the vector $\mathbf{x}_{0}$.

\section{SECOND MOMENTS FOR A COLLECTION OF INITIAL TRAJECTORIES; MATCHING REQUIREMENTS}

Usually, we will be interested in the behavior of a collection of trajectories, corresponding to some initial distribution in phase space. Let us call the initial distribution $f\left(\mathbf{x}_{0}\right)$, and require that it be normalized. Then the average of the matrix $\mathbf{X}_{0}$ over this distribution is

$$
\left\langle x_{0 i} x_{0 j}\right\rangle=\int f\left(\mathbf{x}_{0}\right) x_{0 i} x_{0 j} d \mathbf{x}_{0} .
$$

In this equation and in what follows, the symbol \langle\rangle refers to an average over the initial distribution. We see that, upon averaging over the initial distribution of phase space trajectories, the matrix $\mathbf{X}_{0}$ becomes the matrix of second moments of the initial distribution. To obtain some simple results, we specialize to the case of a cylindrically symmetric initial distribution. In this case, we have

$$
\begin{gathered}
\left\langle x_{0}^{2}\right\rangle=\frac{\beta_{0} \varepsilon_{0}}{2}=\left\langle y_{0}^{2}\right\rangle, \quad\left\langle x_{0} x_{0}^{\prime}\right\rangle=-\frac{\alpha_{0} \varepsilon_{0}}{2}=\left\langle y_{0} y_{0}^{\prime}\right\rangle \\
\left\langle x_{0}^{\prime 2}\right\rangle=\frac{\gamma_{0} \varepsilon_{0}}{2}=\left\langle y_{0}^{\prime 2}\right\rangle, \quad\left\langle x_{0} y_{0}\right\rangle=0=\left\langle x_{0}^{\prime} y_{0}^{\prime}\right\rangle \\
\left\langle x_{0} y_{0}^{\prime}\right\rangle=\frac{L_{0}}{2 P}=-\left\langle x_{0}^{\prime} y_{0}\right\rangle
\end{gathered}
$$

where $\varepsilon_{0}$ is the initial four-dimensional rms geometric emittance of the beam and $L_{0}$ is the initial mean angular momentum of the beam. The averaged initial value matrix is

$$
\left\langle\mathbf{X}_{0}\right\rangle=\left(\begin{array}{cccc}
\frac{\beta_{0} \varepsilon_{0}}{2} & -\frac{\alpha_{0} \varepsilon_{0}}{2} & 0 & \frac{L_{0}}{2 P} \\
-\frac{\alpha_{0} \varepsilon_{0}}{2} & \frac{\gamma_{0} \varepsilon_{0}}{2} & -\frac{L_{0}}{2 P} & 0 \\
0 & -\frac{L_{0}}{2 P} & \frac{\beta_{0} \varepsilon_{0}}{2} & -\frac{\alpha_{0} \varepsilon_{0}}{2} \\
\frac{L_{0}}{2 P} & 0 & -\frac{\alpha_{0} \varepsilon_{0}}{2} & \frac{\gamma_{0} \varepsilon_{0}}{2}
\end{array}\right)
$$

Its determinant is $\operatorname{Det}\left[\left\langle\mathbf{X}_{0}\right\rangle\right]=\frac{\varepsilon_{0}^{4}}{16}\left(1-\rho_{0}^{2}\right)^{2}$, in which $\rho_{0}=\frac{L_{0}}{P \varepsilon_{0}}$. For arbitrary values of $\beta_{0}, \alpha_{0}, \gamma_{0}=\frac{1+\alpha_{0}^{2}}{\beta_{0}}, \varepsilon_{0}$, and $L_{0}$, the elements of the moment matrix,

$$
\langle\mathbf{X}(s)\rangle=\mathbf{T}(s, 0)\left\langle\mathbf{X}_{0}\right\rangle \mathbf{T}^{\mathrm{T}}(s, 0),
$$

will have oscillatory terms proportional to products of $\cos \left(2 \Phi_{0}\right)$ and $\sin \left(2 \Phi_{0}\right)$. These terms correspond to quadrupole oscillations of the beam; they can be made to vanish for the proper choice of $\beta_{0}$ and $\alpha_{0}$. When this choice is satisfied, the input lattice functions are matched to those of the cooling channel. The match conditions, which eliminate these oscillatory terms, are

$$
\begin{aligned}
& 2 \beta_{0}=\frac{\beta_{+}(0)+\beta_{-}(0)}{|C|} \sqrt{4+\left(|C|^{2}-4\right) \rho_{0}^{2}}+\rho_{0}\left[\beta_{+}(0)-\beta_{-}(0)\right], \\
& 2 \alpha_{0}=\frac{\alpha_{+}(0)+\alpha_{-}(0)}{|C|} \sqrt{4+\left(|C|^{2}-4\right) \rho_{0}^{2}}+\rho_{0}\left[\alpha_{+}(0)-\alpha_{-}(0)\right] .
\end{aligned}
$$

For the matched case, the elements of $\langle\mathbf{X}(s)\rangle$ are 


$$
\begin{aligned}
& \left\langle X_{x x}\right\rangle=\left\langle X_{y y}\right\rangle=\frac{1}{\delta}\left(\beta_{+}\left\langle I_{-}\right\rangle+\beta_{-}\left\langle I_{+}\right\rangle\right), \quad\left\langle X_{x x^{\prime}}\right\rangle=\left\langle X_{y y^{\prime}}\right\rangle=-\frac{1}{\delta}\left(\alpha_{+}\left\langle I_{-}\right\rangle+\alpha_{-}\left\langle I_{+}\right\rangle\right), \\
& \left\langle X_{x^{\prime} x^{\prime}}\right\rangle=\left\langle X_{y^{\prime} y^{\prime}}\right\rangle=\frac{1}{\delta}\left(\gamma_{+}\left\langle I_{-}\right\rangle+\gamma_{-}\left\langle I_{+}\right\rangle\right), \quad\left\langle X_{x y^{\prime}}\right\rangle=-\left\langle X_{x^{\prime} y}\right\rangle=\frac{1}{\delta}\left(\left\langle I_{-}\right\rangle-\left\langle I_{+}\right\rangle\right) .
\end{aligned}
$$

In these equations, we have, from Eq. (7.10),

$$
\left\langle I_{ \pm}\right\rangle=\left\langle I_{ \pm 0}\right\rangle \exp \left(-\zeta_{ \pm}\right), \quad\left\langle I_{ \pm 0}\right\rangle=\frac{\varepsilon_{0}\left[\gamma_{0} \beta_{ \pm}(0)+\beta_{0} \gamma_{ \pm}(0)-2 \alpha_{ \pm}(0) \alpha_{0}\right]}{2} \mp \frac{L_{0}}{P} .
$$

The complete second moment matrix is $D_{i j}=\left\langle\overline{x_{i} x_{j}}\right\rangle$, in which the matrix $\mathbf{D}$ is

$$
\mathbf{D}=\mathbf{K}+\langle\mathbf{X}\rangle \text {. }
$$

Explicit values [from Eqs. (8.10) and (9.6)] are

$$
\begin{aligned}
D_{x x} & =D_{y y}=\frac{1}{\delta}\left(\beta_{-}\left\langle\overline{J_{+}}\right\rangle+\beta_{+}\left\langle\overline{J_{-}}\right\rangle-2 \sqrt{\beta_{-} \beta_{+}} J_{c}\right), \\
D_{x x^{\prime}} & =D_{y y^{\prime}}=\frac{1}{\delta}\left[-\alpha_{-}\left\langle\overline{J_{+}}\right\rangle-\alpha_{+}\left\langle\overline{J_{-}}\right\rangle+\left(\alpha_{-} \sqrt{\frac{\beta_{+}}{\beta_{-}}}+\alpha_{+} \sqrt{\frac{\beta_{-}}{\beta_{+}}}\right) J_{c}+\left(\sqrt{\frac{\beta_{+}}{\beta_{-}}}+\sqrt{\frac{\beta_{-}}{\beta_{+}}}\right) J_{s}\right], \\
D_{x^{\prime} x^{\prime}} & =D_{y^{\prime} y^{\prime}}=\frac{1}{\delta}\left[\gamma_{-}\left\langle\overline{J_{+}}\right\rangle+\gamma_{+}\left\langle\overline{J_{-}}\right\rangle+2 \frac{\left(1-\alpha_{+} \alpha_{-}\right)}{\sqrt{\beta_{-} \beta_{+}}} J_{c}-\frac{2\left(\alpha_{+}+\alpha_{-}\right)}{\sqrt{\beta_{-} \beta_{+}}} J_{s}\right], \\
D_{x y} & =D_{x^{\prime} y^{\prime}}=0, \\
D_{x y^{\prime}} & =-D_{x^{\prime} y}=\frac{1}{\delta}\left[\left\langle\overline{J_{-}}\right\rangle-\left\langle\overline{J_{+}}\right\rangle-\left(\alpha_{-} \sqrt{\frac{\beta_{+}}{\beta_{-}}}-\alpha_{+} \sqrt{\frac{\beta_{-}}{\beta_{+}}}\right) J_{s}+\left(\sqrt{\frac{\beta_{+}}{\beta_{-}}}-\sqrt{\frac{\beta_{-}}{\beta_{+}}}\right) J_{c}\right], \\
\sqrt{\operatorname{Det}[\mathbf{D}]} & =D_{x x} D_{x^{\prime} x^{\prime}}-D_{x x^{\prime}}^{2}-D_{x y^{\prime}}^{2}=\frac{1}{\delta}\left[\left\langle\overline{J_{+}}\right\rangle\left\langle\overline{J_{-}}\right\rangle-\left(J_{c}^{2}+J_{s}^{2}\right)\right],
\end{aligned}
$$

in which

$$
\left\langle\overline{J_{ \pm}}\right\rangle=\left\langle I_{ \pm}\right\rangle+J_{ \pm}
$$

The rms four-dimensional geometric emittance $\varepsilon$ is given by

$$
\begin{aligned}
& \varepsilon^{2}=\left(\left\langle\overline{x^{2}+y^{2}}\right\rangle\left\langle\overline{x^{\prime 2}+y^{\prime 2}}\right\rangle-\left\langle\overline{x x^{\prime}+y y^{\prime}}\right\rangle^{2}\right)=4\left(D_{x x} D_{x^{\prime} x^{\prime}}-D_{x x^{\prime}}^{2}\right) \\
&=\frac{4}{\delta^{2}}\left\{\left(\left\langle\overline{J_{-}}\right\rangle+\left\langle\overline{J_{+}}\right\rangle\right)^{2}-\left(J_{s}^{2}+J_{c}^{2}\right)\left(\sqrt{\frac{\beta_{+}}{\beta_{-}}}+\sqrt{\frac{\beta_{-}}{\beta_{+}}}\right)^{2}+\left(\left\langle\overline{J_{+}}\right\rangle\left\langle\overline{J_{-}}\right\rangle-J_{c}^{2}\right)(\delta-4)\right. \\
&\left.\quad+2\left[J_{c}\left(\sqrt{\frac{\beta_{+}}{\beta_{-}}}-\sqrt{\frac{\beta_{-}}{\beta_{+}}}\right)-J_{s}\left(\alpha_{-} \sqrt{\frac{\beta_{+}}{\beta_{-}}}-\alpha_{+} \sqrt{\frac{\beta_{-}}{\beta_{+}}}\right)\right]\left[J_{c}\left(\sqrt{\frac{\beta_{+}}{\beta_{-}}}-\sqrt{\frac{\beta_{-}}{\beta_{+}}}\right)-\left\langle\overline{J_{+}}\right\rangle+\left\langle\overline{J_{-}}\right\rangle\right]\right\} .
\end{aligned}
$$

The mean angular momentum is

$$
\frac{\langle\bar{L}\rangle}{P}=D_{x y^{\prime}}-D_{x^{\prime} y}=\frac{2}{\delta}\left[\left\langle\overline{J_{-}}\right\rangle-\left\langle\overline{J_{+}}\right\rangle+J_{c}\left(\sqrt{\frac{\beta_{+}}{\beta_{-}}}-\sqrt{\frac{\beta_{-}}{\beta_{+}}}\right)-J_{s}\left(\alpha_{-} \sqrt{\frac{\beta_{+}}{\beta_{-}}}-\alpha_{+} \sqrt{\frac{\beta_{-}}{\beta_{+}}}\right)\right] .
$$

\section{DISTRIBUTION FUNCTION FOR AN INITIAL GAUSSIAN DISTRIBUTION OF TRAJECTORIES}

If the initial distribution is taken to be Gaussian,

$$
f\left(\mathbf{x}_{0}\right)=\frac{1}{(2 \pi)^{2} \sqrt{\operatorname{Det}\left[\left\langle\mathbf{X}_{0}\right\rangle\right]}} \exp \left(-\frac{\mathbf{x}_{0}\left\langle\mathbf{X}_{0}\right\rangle^{-1} \mathbf{x}_{0}}{2}\right),
$$

then the distribution function $W\left(\mathbf{x}, \mathbf{x}_{0}, s\right)$, averaged over this initial distribution, also has a Gaussian form. We have 


$$
\begin{aligned}
\langle W(\mathbf{x}, s)\rangle & =\int d \mathbf{x}_{0} f\left(\mathbf{x}_{0}\right) W\left(\mathbf{x}, \mathbf{x}_{0}, s\right) \\
& =\frac{\exp (2 \tilde{n})}{(2 \pi)^{4} \Delta \sqrt{\operatorname{Det}\left[\left\langle\mathbf{X}_{0}\right\rangle\right]}} \int d \mathbf{x}_{0} \exp \left\{-\frac{\left[\mathbf{x}-\mathbf{T}(s, 0) \mathbf{x}_{0}\right] \mathbf{K}^{-1}\left[\mathbf{x}-\mathbf{T}(s, 0) \mathbf{x}_{0}\right]}{2}-\frac{\mathbf{x}_{0}\left\langle\mathbf{X}_{0}\right\rangle^{-1} \mathbf{x}_{0}}{2}\right\} \\
& =\frac{1}{(2 \pi)^{2} \sqrt{\operatorname{Det}[\mathbf{D}]}} \exp \left(-\frac{\mathbf{x} \mathbf{D}^{-1} \mathbf{x}}{2}\right),
\end{aligned}
$$

with $\mathbf{D}$ given by Eq. (9.9). Note that, from Eq. (9.9), we have

$$
\sqrt{\operatorname{Det}[\mathbf{D}]}=\frac{1}{4}\left(\varepsilon^{2}-\frac{\langle\bar{L}\rangle^{2}}{P^{2}}\right) \text {. }
$$

The peak density at the origin of phase space is given by

$$
\langle W(0,0,0,0, s)\rangle=\frac{1}{(2 \pi)^{2} \sqrt{\operatorname{Det}[\mathbf{D}]}}=\frac{\delta}{(2 \pi)^{2}\left[\left\langle\overline{J_{+}}\right\rangle\left\langle\overline{J_{-}}\right\rangle-\left(J_{c}^{2}+J_{s}^{2}\right)\right]} .
$$

The distribution function may be expressed somewhat more simply by using reduced variables. We introduce reduced variables defined by the following linear transformation:

$$
\mathbf{x}=\mathbf{\Omega} \zeta
$$

in which

$$
\boldsymbol{\Omega}=\left(\begin{array}{cccc}
\frac{2 \sqrt{D_{x x}}}{\varepsilon} & 0 & 0 & 0 \\
\frac{2 D_{x x^{\prime}}}{\varepsilon \sqrt{D_{x x}}} & \frac{1}{\sqrt{D_{x x}}} & 0 & 0 \\
0 & 0 & \frac{2 \sqrt{D_{y y}}}{\varepsilon} & 0 \\
0 & 0 & \frac{2 D_{y y^{\prime}}}{\varepsilon \sqrt{D_{y y}}} & \frac{1}{\sqrt{D_{y y}}}
\end{array}\right) .
$$

The quadratic form in Eq. (10.2) becomes

$$
\mathbf{x D}^{-1} \mathbf{x}=\frac{1}{\sqrt{\operatorname{Det}[\mathbf{D}]}}\left[\zeta_{1}^{2}+\zeta_{2}^{2}+\zeta_{3}^{2}+\zeta_{4}^{2}+\frac{4 D_{x y^{\prime}}}{\varepsilon}\left(\zeta_{2} \zeta_{3}-\zeta_{1} \zeta_{4}\right)\right] .
$$

Introducing polar coordinates through the equations

$$
\zeta_{1}=\nu_{x} \cos \phi_{x}, \quad \zeta_{2}=\nu_{x} \sin \phi_{x}, \quad \zeta_{3}=\nu_{y} \cos \phi_{y}, \quad \zeta_{4}=\nu_{y} \sin \phi_{y},
$$

the quadratic form becomes

$$
\mathbf{x D}^{-1} \mathbf{x}=\frac{1}{\sqrt{\operatorname{Det}[\mathbf{D}]}}\left[\nu_{x}^{2}+\nu_{y}^{2}+\frac{4 D_{x y^{\prime}}}{\varepsilon} \nu_{x} \nu_{y} \sin \left(\phi_{x}-\phi_{y}\right)\right] .
$$

The distribution function in polar coordinates is

$$
\begin{aligned}
\left\langle W_{\phi}\left(\nu_{x}, \nu_{y}, \phi_{x}, \phi_{y}, s\right)\right\rangle & =\langle W(\mathbf{x}, s)\rangle|\operatorname{Det}[\mathbf{\Omega}]| \nu_{x} \nu_{y} \\
& =\frac{1}{(2 \pi)^{2} \sqrt{\operatorname{Det}[\mathbf{D}]}} \frac{4 \nu_{x} \nu_{y}}{\varepsilon^{2}} \exp \left(-\frac{\nu_{x}^{2}+\nu_{y}^{2}+\frac{4 D_{x y}{ }^{\prime}}{\varepsilon} \nu_{x} \nu_{y} \sin \left(\phi_{x}-\phi_{y}\right)}{2 \sqrt{\operatorname{Det}[\mathbf{D}]}}\right) .
\end{aligned}
$$

We can integrate this equation over the angular variables to obtain

$$
\left\langle W_{\nu}\left(\nu_{x}, \nu_{y}, s\right)\right\rangle=\int_{0}^{2 \pi} d \phi_{x} \int_{0}^{2 \pi} d \phi_{y}\left\langle W_{\phi}\left(\nu_{x}, \nu_{y}, \phi_{x}, \phi_{y}, s\right)\right\rangle=\frac{1}{\sqrt{\operatorname{Det}[\mathbf{D}]}} \frac{4 \nu_{x} \nu_{y}}{\varepsilon^{2}} \exp \left(-\frac{\nu_{x}^{2}+\nu_{y}^{2}}{2 \sqrt{\operatorname{Det}[\mathbf{D}]}}\right) I_{0}\left(\frac{2 \nu_{x} \nu_{y} D_{x y^{\prime}}}{\varepsilon \sqrt{\operatorname{Det}[\mathbf{D}]}}\right) .
$$

Introducing another set of polar coordinates through

$$
\nu_{x}=r \cos \theta, \quad \nu_{y}=r \sin \theta,
$$

and integrating over the angle $\theta$ gives a distribution function in terms of $r$ :

$$
\left\langle W_{r}(r, s)\right\rangle=\int_{0}^{\pi / 2} d \theta\left\langle W_{\nu}(r \cos \theta, r \sin \theta, s)\right\rangle=\frac{2 r}{\varepsilon D_{x y^{\prime}}} \exp \left(-\frac{r^{2}}{2 \sqrt{\operatorname{Det}[\mathbf{D}]}}\right) \sinh \left(\frac{r^{2} D_{x y^{\prime}}}{\varepsilon \sqrt{\operatorname{Det}[\mathbf{D}]}}\right) .
$$


The variable $r$ is closely related to the four-dimensional emittance. From Eqs. (10.5), (10.8), and (10.12), we have

$$
r^{2}=\nu_{x}^{2}+\nu_{y}^{2}=D_{x^{\prime} x^{\prime}}\left(x^{2}+y^{2}\right)+D_{x x}\left(x^{\prime 2}+y^{\prime 2}\right)-2 D_{x x^{\prime}}\left(x x^{\prime}+y y^{\prime}\right) .
$$

The area in phase space included within the ellipse defined by

$$
\nu_{x}^{2}=D_{x^{\prime} x^{\prime}} x^{2}+D_{x x} x^{\prime 2}-2 D_{x x^{\prime}} x x^{\prime}
$$

is

$$
A_{x}=\frac{\pi \nu_{x}^{2}}{\sqrt{D_{x^{\prime} x^{\prime}} D_{x x}-D_{x x^{\prime}}^{2}}}=\frac{2 \pi \nu_{x}^{2}}{\varepsilon}=\pi \varepsilon_{x} \Rightarrow \nu_{x}^{2}=\frac{\varepsilon_{x} \varepsilon}{2},
$$

in which $\varepsilon_{x}$ is the $\left\{x, x^{\prime}\right\}$ plane emittance. We have a similar relation for the $\left\{y, y^{\prime}\right\}$ plane, so

$$
r^{2}=\nu_{x}^{2}+\nu_{y}^{2}=\frac{\varepsilon_{x} \varepsilon}{2}+\frac{\varepsilon_{y} \varepsilon}{2}=\frac{\varepsilon \varepsilon_{x y}}{2},
$$

in which $\varepsilon_{x y}=\varepsilon_{x}+\varepsilon_{y}$ is the four-dimensional emittance. From Eqs. (10.13) and (10.17), the distribution function in the four-dimensional emittance is

$$
\left\langle W_{\varepsilon}\left(\varepsilon_{x y}, s\right)\right\rangle=\left[\left\langle W_{r}(r, s)\right\rangle \frac{\varepsilon}{4 r}\right]_{r=\sqrt{\varepsilon \varepsilon_{x y} / 2}}=\frac{1}{2 D_{x y^{\prime}}} \exp \left(-\frac{\varepsilon \varepsilon_{x y}}{4 \sqrt{\overline{\operatorname{Det}[\mathbf{D}]}}}\right) \sinh \left(\frac{\varepsilon_{x y} D_{x y^{\prime}}}{2 \sqrt{\operatorname{Det}[\mathbf{D}]}}\right) .
$$

The fraction of the beam contained within the total four-dimensional emittance $\varepsilon_{x y} \leq \varepsilon_{\text {max }}$ may be obtained by integrating Eq. (10.18):

$$
f\left(\varepsilon_{\max }, s\right)=\int_{0}^{\varepsilon_{\max }}\left\langle W_{\varepsilon}\left(\varepsilon_{x y}, s\right)\right\rangle d \varepsilon_{x y}=1-\exp \left(-\frac{\varepsilon \varepsilon_{\max }}{4 \sqrt{\overline{\operatorname{Det}}[\mathbf{D}]}}\right)\left[\cosh \left(\frac{\varepsilon_{\max } D_{x y^{\prime}}}{2 \sqrt{\operatorname{Det}[\mathbf{D}]}}\right)+\frac{\varepsilon}{2 D_{x y^{\prime}}} \sinh \left(\frac{\varepsilon_{\max } D_{x y^{\prime}}}{2 \sqrt{\operatorname{Det}[\mathbf{D}]}}\right)\right] .
$$

\section{PERIODIC SYSTEMS}

We now consider axisymmetric systems for which the quantities $k, \eta$, and $G$ are all periodic with period $\lambda$. Equation (5.6) must then be solved for the lattice functions, which are required to satisfy periodic boundary conditions

$$
\beta_{ \pm}(0)=\beta_{ \pm}(\lambda), \quad \beta_{ \pm}^{\prime}(0)=\beta_{ \pm}^{\prime}(\lambda) .
$$

In general, certain stability criteria must be met for periodic solutions to be found [12]. We assume that these conditions are satisfied.

\section{A. General relations for periodic systems}

We want to develop a formula for the elements of the $\mathbf{D}$ matrix after $n$ periods. We start with Eq. (5.12), which can be written as

$$
\zeta_{ \pm}(s+n \lambda)=\int_{0}^{s+n \lambda} \eta\left(s^{\prime}\right)\left[1 \pm \beta_{ \pm}\left(s^{\prime}\right) k\left(s^{\prime}\right)\right] d s^{\prime}=n \zeta_{ \pm}(\lambda)+\zeta_{ \pm}(s)
$$

Similarly, we have for Eq. (5.5)

$\Phi_{ \pm}(s+n \lambda)=\int_{0}^{s+n \lambda} \frac{d s^{\prime}}{\beta_{ \pm}\left(s^{\prime}\right)}=\int_{0}^{n \lambda} \frac{d s^{\prime}}{\beta_{ \pm}\left(s^{\prime}\right)}+\int_{n \lambda}^{s+n \lambda} \frac{d s^{\prime}}{\beta_{ \pm}\left(s^{\prime}\right)}=n \mu_{ \pm}+\int_{0}^{s} \frac{d s^{\prime}}{\beta_{ \pm}\left(s^{\prime}\right)}=n \mu_{ \pm}+\Phi_{ \pm}(s)$,

in which

$$
\mu_{ \pm}=\int_{0}^{\lambda} \frac{d s^{\prime}}{\beta_{ \pm}\left(s^{\prime}\right)}
$$

is the phase advance per period. For Eq. (7.9), we have

$$
\begin{aligned}
J_{ \pm}(n \lambda) & =\exp \left[-\zeta_{ \pm}(n \lambda)\right] \int_{0}^{n \lambda} G\left(s^{\prime}\right)^{2} \beta_{ \pm}\left(s^{\prime}\right) \exp \left[\zeta_{ \pm}\left(s^{\prime}\right)\right] d s^{\prime}=\exp \left[-n \zeta_{ \pm}(\lambda)\right] \sum_{m=0}^{n-1} \int_{m \lambda}^{(m+1) \lambda} G\left(s^{\prime}\right)^{2} \beta_{ \pm}\left(s^{\prime}\right) \exp \left[\zeta_{ \pm}\left(s^{\prime}\right)\right] d s^{\prime} \\
& =\exp \left[-n \zeta_{ \pm}(\lambda)\right] \sum_{m=0}^{n-1} \int_{0}^{\lambda} G\left(s^{\prime}+m \lambda\right)^{2} \beta_{ \pm}\left(s^{\prime}+m \lambda\right) \exp \left[\zeta_{ \pm}\left(s^{\prime}+m \lambda\right)\right] d s^{\prime}
\end{aligned}
$$


Using Eq. (11.2) and the periodicity of $\beta_{ \pm}$and $G$, Eq. (11.5) becomes

$$
\begin{aligned}
J_{ \pm}(n \lambda) & =\exp \left[-n \zeta_{ \pm}(\lambda)\right] \int_{0}^{\lambda} G\left(s^{\prime}\right)^{2} \beta_{ \pm}\left(s^{\prime}\right) \exp \left[\zeta_{ \pm}\left(s^{\prime}\right)\right] \sum_{m=0}^{n-1} \exp \left[m \zeta_{ \pm}(\lambda)\right] \\
& =J_{ \pm}(\lambda) \exp \left[-(n-1) \zeta_{ \pm}(\lambda)\right] \sum_{m=0}^{n-1} \exp \left[m \zeta_{ \pm}(\lambda)\right] .
\end{aligned}
$$

Using the theorem

$$
\exp [-(n-1) z] \sum_{m=0}^{n-1} \exp (m z)=\frac{1-\exp (-n z)}{1-\exp (-z)}
$$

Eq. (11.6) becomes

$$
J_{ \pm}(n \lambda)=J_{ \pm}(\lambda) \frac{1-\exp \left[-n \zeta_{ \pm}(\lambda)\right]}{1-\exp \left[-\zeta_{ \pm}(\lambda)\right]}
$$

For Eq. (7.10), we have

$$
I_{ \pm}(n \lambda)=I_{ \pm 0} \exp \left[-n \zeta_{ \pm}(\lambda)\right]
$$

For Eq. (8.11), we have

$$
\begin{aligned}
J_{c}(n \lambda)+i J_{s}(n \lambda)= & \exp \left[-\zeta_{0}(n \lambda)+2 i \Phi_{0}(n \lambda)\right] \int_{0}^{n \lambda} G\left(s^{\prime}\right)^{2} \sqrt{\beta_{+}\left(s^{\prime}\right) \beta_{-}\left(s^{\prime}\right)} \exp \left[\zeta_{0}\left(s^{\prime}\right)-2 i \Phi_{0}\left(s^{\prime}\right)\right] d s^{\prime} \\
= & \exp \left[-\zeta_{0}(n \lambda)+2 i \Phi_{0}(n \lambda)\right] \\
& \times \sum_{m=0}^{n-1} \int_{0}^{\lambda} G\left(s^{\prime}+m \lambda\right)^{2} \sqrt{\beta_{+}\left(s^{\prime}+m \lambda\right) \beta_{-}\left(s^{\prime}+m \lambda\right)} \exp \left[\zeta_{0}\left(s^{\prime}+m \lambda\right)-2 i \Phi_{0}\left(s^{\prime}+m \lambda\right)\right] d s^{\prime}
\end{aligned}
$$

From Eqs. (11.2) and (11.3), we have

$$
\begin{aligned}
\exp \left[-\zeta_{0}(n \lambda)+2 i \Phi_{0}(n \lambda)\right] & =\exp \left[-n \zeta_{0}(\lambda)+i n\left(\mu_{+}+\mu_{-}\right)\right], \\
\exp \left[\zeta_{0}\left(s^{\prime}+m \lambda\right)-2 i \Phi_{0}\left(s^{\prime}+m \lambda\right)\right] & =\exp \left[m \zeta_{0}(\lambda)-i m\left(\mu_{+}+\mu_{-}\right)+\zeta_{0}\left(s^{\prime}\right)-2 i \Phi_{0}\left(s^{\prime}\right)\right] .
\end{aligned}
$$

So, Eq. (11.10) becomes

$$
\begin{aligned}
J_{c}(n \lambda)+i J_{s}(n \lambda)= & \exp \left\{n\left[-\zeta_{0}(\lambda)+i\left(\mu_{+}+\mu_{-}\right)\right]\right\} \sum_{m=0}^{n-1} \exp \left[m \zeta_{0}(\lambda)-i m\left(\mu_{+}+\mu_{-}\right)\right] \\
& \times \int_{0}^{\lambda} G\left(s^{\prime}\right)^{2} \sqrt{\beta_{+}\left(s^{\prime}\right) \beta_{-}\left(s^{\prime}\right)} \exp \left[\zeta_{0}\left(s^{\prime}\right)-2 i \Phi_{0}\left(s^{\prime}\right)\right] d s^{\prime} \\
= & {\left[J_{c}(\lambda)+i J_{s}(\lambda)\right] \exp \left\{-(n-1)\left[\zeta_{0}(\lambda)-i\left(\mu_{+}+\mu_{-}\right)\right]\right\} \sum_{m=0}^{n-1} \exp \left\{m\left[\zeta_{0}(\lambda)-i\left(\mu_{+}+\mu_{-}\right)\right]\right\} }
\end{aligned}
$$

Using Eq. (11.7) again gives

$$
J_{c}(n \lambda)+i J_{s}(n \lambda)=\left[J_{c}(\lambda)+i J_{s}(\lambda)\right] \frac{1-\exp \left[i n\left(\mu_{+}+\mu_{-}\right)-n \zeta_{0}(\lambda)\right]}{1-\exp \left[i\left(\mu_{+}+\mu_{-}\right)-\zeta_{0}(\lambda)\right]} .
$$

Let us define

$$
n_{ \pm}=\zeta_{ \pm}(\lambda)
$$

These quantities are the damping rates per period. After $n$ periods, we have for the pseudoinvariants

$$
\begin{aligned}
\left\langle\overline{J_{ \pm}}\right\rangle & =\left\langle I_{ \pm}(n \lambda)\right\rangle+J_{ \pm}(n \lambda)=\left\langle I_{ \pm 0}\right\rangle \exp \left[-n \zeta_{ \pm}(\lambda)\right]+J_{ \pm}(\lambda) \frac{1-\exp \left[-n \zeta_{ \pm}(\lambda)\right]}{1-\exp \left[-\zeta_{ \pm}(\lambda)\right]} \\
& =\exp \left(-n n_{ \pm}\right)\left[\left\langle I_{ \pm 0}\right\rangle-\frac{J_{ \pm}(\lambda)}{1-\exp \left(-n_{ \pm}\right)}\right]+\frac{J_{ \pm}(\lambda)}{1-\exp \left(-n_{ \pm}\right)} .
\end{aligned}
$$

From Eqs. (11.13) and (11.15), the elements of the matrix D [Eq. (9.9)] after $n$ periods may be evaluated. 
Provided that $n_{ \pm}>0,\left\langle\overline{J_{ \pm}}\right\rangle$will approach limits for $n \gg 1 / n_{ \pm}$. Those limits are

$$
\left\langle\overline{\boldsymbol{J}_{ \pm}}\right\rangle_{\infty}=\frac{J_{ \pm}(\lambda)}{1-\exp \left(-n_{ \pm}\right)} .
$$

If $n_{0}=\zeta_{0}(\lambda)>0$, then $J_{c}+i J_{s}$ will also approach a limit for $n \gg 1 / n_{0}$. In this case,

$$
\left(J_{c}+i J_{s}\right)_{\infty}=\frac{J_{c}(\lambda)+i J_{s}(\lambda)}{1-\exp \left[i\left(\mu_{+}+\mu_{-}\right)-n_{0}\right]} .
$$

\section{B. Approximations}

We now consider some simplifying approximations. If we take $n_{ \pm} \ll 1$, then the asymptotic limits simplify to

$$
\begin{aligned}
\left\langle\overline{J_{ \pm}}\right\rangle_{\infty} & \approx \frac{J_{ \pm}(\lambda)}{n_{ \pm}}, \\
\left(J_{c}\right)_{\infty} & \approx \frac{J_{c}(\lambda)-J_{s}(\lambda) \cot \left(\frac{\mu_{+}+\mu_{-}}{2}\right)}{2}, \\
\left(J_{s}\right)_{\infty} & \approx \frac{J_{s}(\lambda)+J_{c}(\lambda) \cot \left(\frac{\mu_{+}+\mu_{-}}{2}\right)}{2} .
\end{aligned}
$$

Since $J_{ \pm}(\lambda)>J_{c}(\lambda) \approx J_{s}(\lambda)$, for $n_{ \pm} \ll 1$, the emittance approaches the limit

$$
\begin{aligned}
\varepsilon_{\infty} & \approx \frac{2}{\delta(0)}\left(\left\langle\overline{\boldsymbol{J}_{+}}\right\rangle_{\infty}+\left\langle\overline{\boldsymbol{J}_{-}}\right\rangle_{\infty}\right) \\
& \approx \frac{1}{2}\left(\frac{J_{+}(\lambda)}{n_{+}}+\frac{J_{-}(\lambda)}{n_{-}}\right),
\end{aligned}
$$

in which we used $\delta(0) \approx 4$. The peak density approaches the limit

$$
\langle W(0,0,0,0, \infty)\rangle \approx \frac{1}{\pi^{2}} \frac{n_{+} n_{-}}{J_{+}(\lambda) J_{-}(\lambda)} .
$$

\section{Special case}

A special case that often occurs in practice is worth commenting on. If the electric and magnetic fields have the property that

$$
k(s)=-k(\lambda-s), \quad \eta(s)=\eta(\lambda-s),
$$

then, from Eq. (5.6), we have

$$
\beta_{ \pm}(s)=\beta_{ \pm}(\lambda-s) .
$$

In this case, there is only one damping rate:

$$
\int_{0}^{\lambda} \eta\left(s^{\prime}\right) \beta_{ \pm}\left(s^{\prime}\right) k\left(s^{\prime}\right) d s^{\prime}=0 \Rightarrow \zeta_{+}(\lambda)=\zeta_{-}(\lambda)=\int_{0}^{\lambda} \eta\left(s^{\prime}\right) d s^{\prime}=\tilde{n}(\lambda),
$$

and, for $n_{+}=n_{-} \ll 1$, the emittance and peak density approach the limits

$$
\begin{aligned}
\varepsilon_{\infty} & \approx \frac{\int_{0}^{\lambda} G\left(s^{\prime}\right)^{2}\left[\beta_{+}\left(s^{\prime}\right)+\beta_{-}\left(s^{\prime}\right)\right] d s^{\prime}}{2 \tilde{n}(\lambda)}, \\
\langle W(0,0,0,0, \infty)\rangle & \approx \frac{1}{\pi^{2}} \frac{\tilde{n}(\lambda)^{2}}{\left[\int_{0}^{\lambda} G\left(s^{\prime}\right)^{2} \beta_{+}\left(s^{\prime}\right) d s^{\prime}\right]\left[\int_{0}^{\lambda} G\left(s^{\prime}\right)^{2} \beta_{-}\left(s^{\prime}\right) d s^{\prime}\right]} .
\end{aligned}
$$

Note that, in this case, we have from Eq. (4.14):

$$
n_{+}=n_{-}=\tilde{n}(\lambda)=\frac{\Delta \mathcal{E}}{P \beta c},
$$

in which $\Delta \mathcal{E}$ is the energy loss per period. Using Eq. (3.9), the asymptotic normalized four-dimensional emittance is given by

$$
\varepsilon_{\infty, \text { norm }}=\beta \gamma \varepsilon_{\infty} \approx \frac{(13.6 \mathrm{MeV})^{2}}{2 \beta(\Delta \mathcal{E}) m c^{2}} \frac{\int_{0}^{L_{a}}\left[\beta_{+}\left(s^{\prime}\right)+\beta_{-}\left(s^{\prime}\right)\right] d s^{\prime}}{L_{\mathrm{rad}}},
$$

in which $L_{a}$ is the length of the absorber, in which the multiple scattering takes place. The peak density is

$$
\langle W(0,0,0,0, \infty)\rangle \approx \frac{1}{\pi^{2}} \frac{(\Delta \mathcal{E})^{2}(P \beta c)^{2}}{(13.6 \mathrm{MeV})^{4}} \frac{L_{\mathrm{rad}}^{2}}{\left[\int_{0}^{L_{a}} \beta_{+}\left(s^{\prime}\right) d s^{\prime}\right]\left[\int_{0}^{L_{a}} \beta_{-}\left(s^{\prime}\right) d s^{\prime}\right]} .
$$

\section{RELATION OF THIS WORK TO REF. [3]}

We note that, associated with stochastic differential equations of the form of Eq. (6.1), there is a set of moment equations for the general dynamical variable $A$ which have the form (for $\mathbf{Q}$ independent of $\mathbf{x}$, as in this case)

$$
\frac{d}{d s} \bar{A}=\sum_{i} K_{i} \frac{\overline{\partial A}}{\partial x_{i}}+\frac{1}{2} \sum_{i, j, k} Q_{i k} Q_{k j} \frac{\overline{\partial^{2} A}}{\partial x_{i} \partial x_{j}} .
$$


For this application, and for various choices of $A$, we have, using Eq. (6.4),

$$
\begin{aligned}
\frac{d}{d s}\left\langle\overline{x^{2}+y^{2}}\right\rangle & =2\left\langle\overline{x x^{\prime}+y y^{\prime}}\right\rangle \\
\frac{d}{d s}\left\langle\overline{x^{\prime 2}+y^{\prime 2}}\right\rangle & \left.=-\left\langle\overline{x x^{\prime}+y y^{\prime}}\right\rangle\left(2 k^{2}+\eta^{\prime}+\Pi \eta\right)-2 \eta\left(\overline{\left\langle x^{\prime 2}+y^{\prime 2}\right.}\right\rangle-k \frac{\langle\bar{L}\rangle}{P}\right)+2 G^{2}, \\
\frac{d}{d s}\left\langle\overline{x x^{\prime}+y y^{\prime}}\right\rangle & =-\left\langle\overline{x x^{\prime}+y y^{\prime}}\right\rangle \eta-\left\langle\overline{x^{2}+y^{2}}\right\rangle\left[k^{2}+\frac{1}{2}\left(\eta^{\prime}+\Pi \eta\right)\right]+\left\langle\overline{x^{\prime 2}+y^{\prime 2}}\right\rangle, \\
\frac{d}{d s}\left\langle\overline{x y^{\prime}-y x^{\prime}}\right\rangle & =\frac{d}{d s} \frac{\langle\bar{L}\rangle}{P}=\eta\left(k\left\langle\overline{x^{2}+y^{2}}\right\rangle-\frac{\langle\bar{L}\rangle}{P}\right) .
\end{aligned}
$$

These are the same moment equations as given in Ref. [3], except for (i) the terms in $\eta^{\prime}+\Pi \eta$, an effect which was not treated in Ref. [3], and (ii) a factor of 2 in the coefficient of $G^{2}$ in the second line of Eq. (12.2).

In Ref. [3], the effect of the electric field on the lattice functions was neglected. If we make this approximation here, we then have

$$
\beta_{+}(s)=\beta_{-}(s)=\beta(s), \quad \alpha_{+}(s)=\alpha_{-}(s)=\alpha(s) .
$$

From Eq. (5.14), $\delta$ is independent of $s$ :

$$
\delta(s)=\delta(0)=\left\{2\left[1-\alpha(0)^{2}\right]+2 \beta(0) \gamma(0)\right\}=4 .
$$

The match condition for a cylindrically symmetric initial distribution is just

$$
\beta_{0}=\beta(0), \quad \alpha_{0}=\alpha(0) .
$$

From Eq. (9.9), the elements of the covariance matrix are

$$
\begin{aligned}
D_{x x} & =D_{y y}=\frac{\beta}{4}\left(I_{-}+J_{-}+I_{+}+J_{+}-2 J_{c}\right), \\
D_{x x^{\prime}} & =D_{y y^{\prime}}=\frac{1}{4}\left[-\alpha\left(I_{-}+J_{-}+I_{+}+J_{+}\right)+2\left(J_{s}+\alpha J_{c}\right)\right], \\
D_{x^{\prime} x^{\prime}} & =D_{y^{\prime} y^{\prime}}=\frac{1}{4}\left[\gamma\left(I_{-}+J_{-}+I_{+}+J_{+}+2 J_{c}\right)-\frac{4 \alpha}{\beta} J_{s}\right], \\
D_{x y} & =D_{x^{\prime} y^{\prime}}=0 \\
D_{x y^{\prime}} & =-D_{x^{\prime} y}=\frac{1}{4}\left(I_{-}+J_{-}-I_{+}-J_{+}\right)
\end{aligned}
$$

in which

$$
\begin{aligned}
I_{ \pm} & =\exp \left(-\zeta_{ \pm}\right)\left(\varepsilon_{0} \mp \frac{L_{0}}{P}\right), \\
J_{ \pm} & =\exp \left(-\zeta_{ \pm}\right) \int_{0}^{s} G\left(s^{\prime}\right)^{2} \beta\left(s^{\prime}\right) \exp \left[\zeta_{ \pm}\left(s^{\prime}\right)\right] d s^{\prime}, \\
J_{c}+i J_{s} & =\exp (-\tilde{n}+2 i \Phi) \int_{0}^{s} G\left(s^{\prime}\right)^{2} \beta\left(s^{\prime}\right) \exp \left[\tilde{n}\left(s^{\prime}\right)-2 i \Phi\left(s^{\prime}\right)\right] d s^{\prime}, \\
\zeta_{ \pm}(s) & =\int_{0}^{s} d s^{\prime} \eta\left(s^{\prime}\right)\left[1 \pm k\left(s^{\prime}\right) \beta\left(s^{\prime}\right)\right] .
\end{aligned}
$$

The rms emittance and mean angular momentum are given by 


$$
\begin{gathered}
\varepsilon^{2}=\frac{1}{4}\left(\exp \left(-\zeta_{+}\right)\left\{\varepsilon_{0}-\frac{L_{0}}{P}+\int_{0}^{s} G\left(s^{\prime}\right)^{2} \beta\left(s^{\prime}\right) \exp \left[\zeta_{+}\left(s^{\prime}\right)\right] d s^{\prime}\right\}\right. \\
\left.+\exp \left(-\zeta_{-}\right)\left\{\varepsilon_{0}+\frac{L_{0}}{P}+\int_{0}^{s} G\left(s^{\prime}\right)^{2} \beta\left(s^{\prime}\right) \exp \left[\zeta_{-}\left(s^{\prime}\right)\right] d s^{\prime}\right\}\right)^{2} \\
-\exp (-2 \tilde{n})\left(\left[\int_{0}^{s} G\left(s^{\prime}\right)^{2} \beta\left(s^{\prime}\right) \exp \left[\tilde{n}\left(s^{\prime}\right)\right] \cos \left\{2\left[\Phi(s)-\Phi\left(s^{\prime}\right)\right]\right\} d s^{\prime}\right]^{2}\right. \\
\left.+\left[\int_{0}^{s} G\left(s^{\prime}\right)^{2} \beta\left(s^{\prime}\right) \exp \left[\tilde{n}\left(s^{\prime}\right)\right] \sin \left\{2\left[\Phi(s)-\Phi\left(s^{\prime}\right)\right]\right\} d s^{\prime}\right]^{2}\right) \\
\frac{\langle\bar{L}\rangle}{P}=\frac{1}{2}\left(\exp \left(-\zeta_{-}\right)\left\{\varepsilon_{0}+\frac{L_{0}}{P}+\int_{0}^{s} G\left(s^{\prime}\right)^{2} \beta\left(s^{\prime}\right) \exp \left[\zeta_{-}\left(s^{\prime}\right)\right] d s^{\prime}\right\}\right. \\
\left.-\exp \left(-\zeta_{+}\right)\left\{\varepsilon_{0}-\frac{L_{0}}{P}+\int_{0}^{s} G\left(s^{\prime}\right)^{2} \beta\left(s^{\prime}\right) \exp \left[\zeta_{+}\left(s^{\prime}\right)\right] d s^{\prime}\right\}\right)
\end{gathered}
$$

These equations are the same as the expressions given in Ref. [3], except for the presence of the last (oscillatory) term in the emittance equation.

\section{NUMERICAL EXAMPLE}

It is instructive to treat a numerical example using this formalism. An example that is well characterized and has been studied using numerical simulations is the base line FOFO ionization cooling channel designed for the Fermilab Neutrino Source study [8].

This design uses a periodically varying solenoidal magnetic field, given by

$$
B(s)=B_{0} \sin \left(\frac{2 \pi s}{\lambda}\right),
$$

and an electromagnetic field provided by $201 \mathrm{MHz}$ rf cavities. The ionization energy loss and multiple scattering take place in liquid hydrogen $\left(\mathrm{LH}_{2}\right)$ absorbers. The important parameters of the design are listed in Table I, taken from Ref. [8].
In Fig. 1, we show the model taken for the energy loss and electric field, over one period of the structure. The energy loss takes place in absorbers located at the start and end of the section. The energy is restored by an rf cavity, centered on the middle of the section, with parameters as given in Table I. The accelerating field just restores the energy lost by the absorbers; the roll-off in $s$ at the ends of the cavity has been taken appropriate to the radial aperture $(17 \mathrm{~cm})$.

In Fig. 2, we show the functions $k(s)$ and $\eta(s)$ over one period (two sections).

In Fig. 3, the lattice functions $\beta_{ \pm}(s)$ are shown, over one period, obtained by a numerical solution to Eq. (5.6), with the periodic boundary conditions specified in Eq. (11.1). Figure 4 plots the difference between $\beta_{+}$and $\beta_{-}$, divided

\begin{tabular}{|c|c|c|}
\hline Input parameter & Symbol & Value \\
\hline Section length $=$ one-half period & $\lambda / 2$ & $1.1 \mathrm{~m}$ \\
\hline Maximum magnetic field on axis & $B_{0}$ & $3.4 \mathrm{~T}$ \\
\hline Absorber $\mathrm{LH}_{2}$ scattering parameter & $G^{2}$ & $6.8 \times 10^{-4} / \mathrm{m}$ \\
\hline Absorber $\mathrm{LH}_{2}$ thickness, per section & $L_{a}$ & Secs. 1-40: $12.6 \mathrm{~cm} ;$ Secs. $>40: 13.2 \mathrm{~cm}$ \\
\hline Absorber Al window scattering parameter & $G^{2}$ & $6.62 \times 10^{-2} / \mathrm{m}$ \\
\hline Absorber Al window thickness, per section & $L_{a}$ & Secs. 1-40: $400 \mu \mathrm{m}$; Secs. $>$ 40: $200 \mu \mathrm{m}$ \\
\hline \multicolumn{3}{|l|}{ Energy loss per section } \\
\hline$=$ acceleration energy gain per section & $\Delta \mathcal{E} / 2$ & $4 \mathrm{MeV}$ \\
\hline Synchronous phase & $\phi_{s}$ & $30^{\circ}$ \\
\hline Linac length, per section & $L_{\mathrm{rf}}$ & $0.66 \mathrm{~m}$ \\
\hline Muon beam momentum & $P=m_{0} c \beta \gamma$ & $200 \mathrm{MeV} / c$ \\
\hline Initial transverse normalized 2D emittance & $\varepsilon_{0, \text { norm }, 2 \mathrm{D}}$ & $15 \mathrm{~mm} \mathrm{rad}$ \\
\hline Initial transverse geometric 4D emittance & $\varepsilon_{0}=2 \varepsilon_{0, \text { norm }, 2 \mathrm{D}} / \beta \gamma$ & $15.75 \mathrm{~mm} \mathrm{rad}$ \\
\hline Initial peak transverse $4 \mathrm{D}$ density & $\langle W(0,0,0,0,0)\rangle$ & $408(\mathrm{~m} \mathrm{rad})^{-2}$ \\
\hline
\end{tabular}
by the average; the relative differences are roughly $\pm 6 \%$.

Since Eq. (11.21) is satisfied for this design, there is only one damping rate, which is given in Table II. The input distribution has a transverse emittance given in Table I, and is assumed to have no initial angular momentum.

TABLE I. Cooling channel and input beam parameters. 


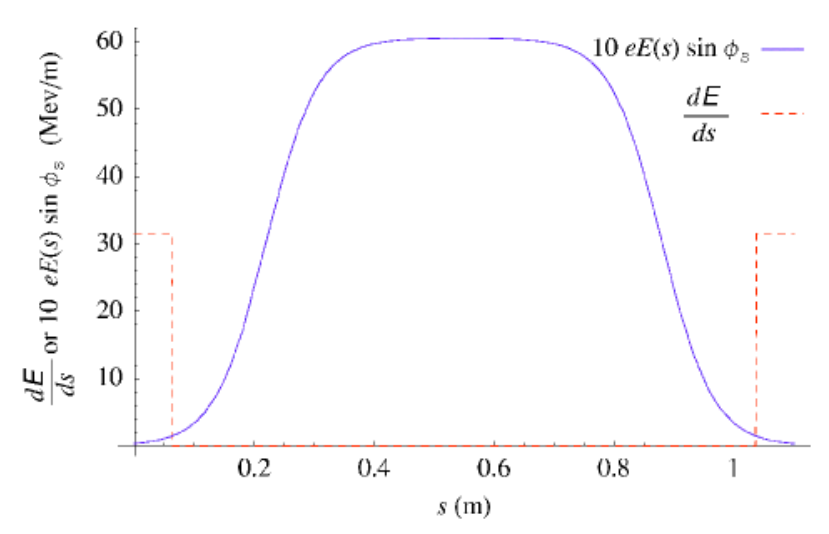

FIG. 1. (Color) Model for the energy loss $d \mathcal{E} / d s$ and accelerating electric field $E(s) \sin \phi_{s}$ over one section.

The lattice parameters required to match into the channel, calculated from Eq. (9.5), are given in Table II.

Also given in Table II are the Wronskian constant for the lattice, the phase advance per period, the multiple scattering integrals, and the asymptotic values of the geometric four-dimensional emittance and the peak transverse four-dimensional density [from Eqs. (11.19) and (11.20)].

In Fig. 5, the evolution of the normalized twodimensional emittance, and mean angular momentum, as a function of distance along the channel, are plotted. In the design of Ref. [8], the channel has 150 sections (75 periods). Figure 5 may be compared with Fig. 7b of Ref. [8], which presents the result of a numerical simulation for the "idealized" beam with parameters given in Table I. There is good agreement. The normalized two-dimensional emittance after 75 periods $(165 \mathrm{~m})$, from Fig. 5, is $2.3 \mathrm{~mm} \mathrm{rad}$, to be compared with a value of $2.2 \mathrm{~mm}$ rad quoted in Ref. [8].

The normalized peak density, that is,

$$
W_{0, \text { norm }}(s)=\frac{\langle W(0,0,0,0, s)\rangle}{\langle W(0,0,0,0, \infty)\rangle},
$$

from Eqs. (10.4) and (11.20), is plotted as a function of distance along the channel in Fig. 6. The figure shows that the normalized peak density continues to grow even

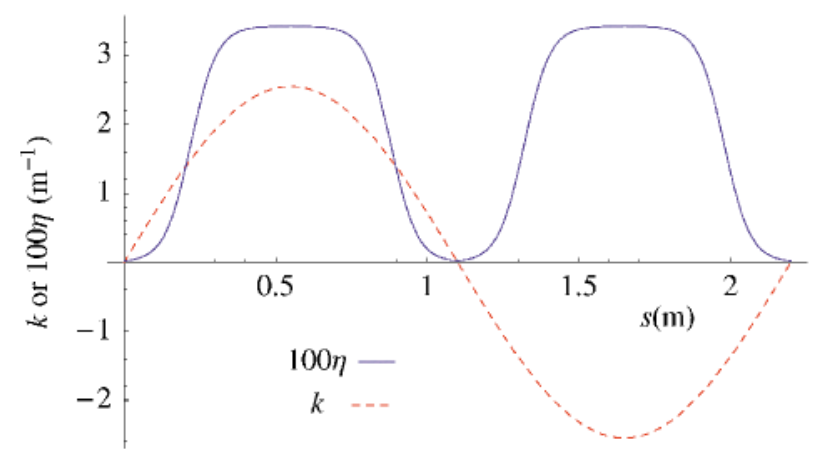

FIG. 2. (Color) The functions $k(s)$ and $\eta(s)$, over one period.

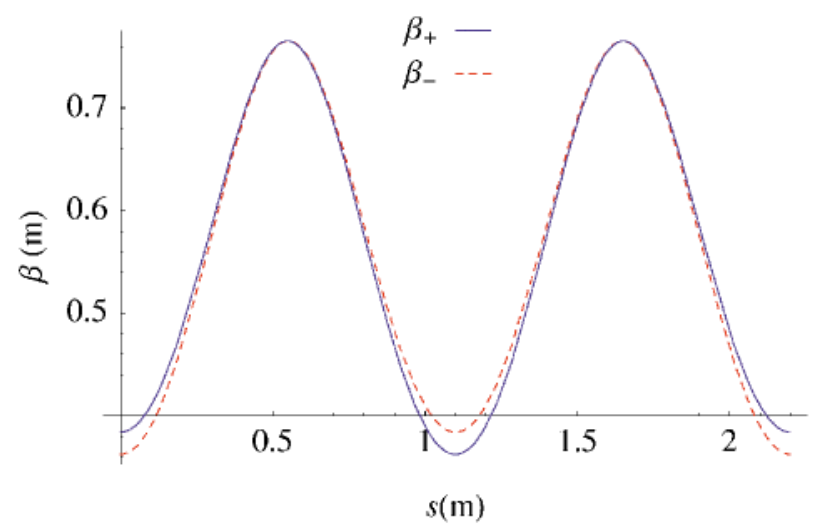

FIG. 3. (Color) The lattice functions $\beta_{ \pm}(s)$, over one period.

beyond $200 \mathrm{~m}$. Also plotted in Fig. 6 is the fraction of the beam with $\varepsilon_{x y}<9.8 \mathrm{~mm}$ rad (geometric four-dimensional emittance), from Eq. (10.19), for comparison with Fig. 7c in Ref. [8]. The fraction with $\varepsilon_{x y}<9.8 \mathrm{~mm}$ rad increases by a factor of 6.3 after $120 \mathrm{~m}$ in this calculation versus a factor of 4.5 quoted in Ref. [8]. The difference may be related to longitudinal losses, which are not considered here but were included in Ref. [8].

In Fig. 7, the density distribution in the fourdimensional emittance $\left(\varepsilon_{x y}\right)$, Eq. (10.18), is plotted as a function of $\varepsilon_{x y}$ at intervals of five periods along the channel, from period 0 to period 75 . The plots are color coded according to the spectrum, varying from red to blue as the period number varies from 0 to 75 .

Finally, in this numerical example, we may estimate the accuracy of the approximations made in Sec. IV to obtain a linear problem. The paraxial approximation assumes that

$$
l^{\prime}=\sqrt{1+x^{\prime 2}+y^{\prime 2}} \approx 1
$$

An estimate of the error made in this assumption can be obtained by calculating

$$
\Delta l^{\prime}=\sqrt{1+\left\langle x^{\prime 2}\right\rangle+\left\langle y^{\prime 2}\right\rangle}-1=\sqrt{1+2 D_{x^{\prime} x^{\prime}}}-1 .
$$

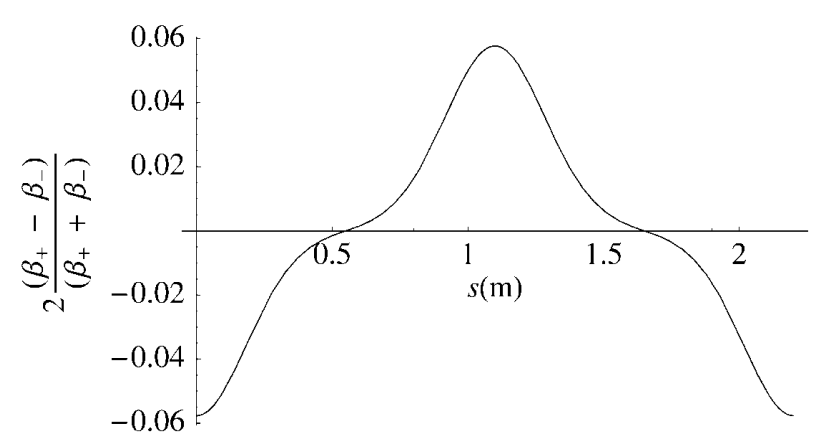

FIG. 4. Relative difference in lattice functions. 
TABLE II. Calculated parameters of cooling channel and cooled beam.

\begin{tabular}{lcc}
\hline \hline \multicolumn{1}{c}{ Calculated parameter } & Symbol & Value \\
\hline Mean number of periods for damping & $n_{+}^{-1}=n_{-}^{-1}$ & 22.1 \\
Phase advances per period & $\left\{\mu_{+}, \mu_{-}\right\}$ & $\{4.13154,4.13155\}$ \\
Matched value of initial beta function & $\beta_{0}$ & $0.373 \mathrm{~m}$ \\
Matched value of initial alpha function & $\alpha_{0}$ & $38 \times 10^{-6}$ \\
Wronskian & $|C|$ & 2.00083 \\
Multiple scattering integrals & $\left\{J_{+}(\lambda), J_{-}(\lambda)\right\}$ & Secs. $1-40:\{104.1,100.8\} \mu \mathrm{m} ;$ \\
& & Secs. $>40:\{87.3,84.6\} \mu \mathrm{m}$ \\
Multiple scattering integrals & $\left\{J_{c}(\lambda), J_{s}(\lambda)\right\}$ & Secs. $1-40:\{-8.0,-15.3\} \mu \mathrm{m} ;$ \\
Asymptotic transverse 4D geometric emittance & $\varepsilon_{\infty}$ & Secs. $>40:\{-6.6,-12.6\} \mu \mathrm{m}$ \\
Asymptotic peak transverse 4D density & $\langle W(0,0,0,0, \infty)\rangle$ & $1.902 \mathrm{~mm} \mathrm{rad}$ \\
\hline \hline
\end{tabular}

A plot of this is shown in Fig. 8 .

We see that the maximum value, obtained at the start to the channel, where the emittance is largest, is only a few percent. Numerical estimates of the other approximations made in Sec. IV [neglecting higher order terms in the field

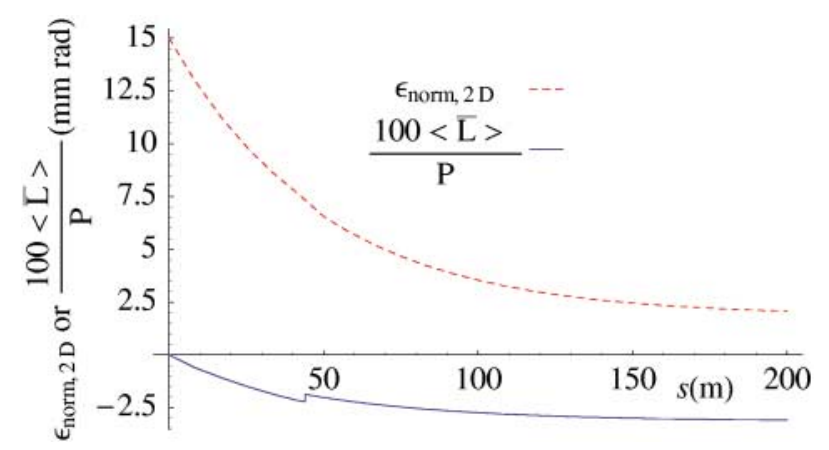

FIG. 5. (Color) Normalized two-dimensional emittance, and angular momentum, as a function of distance along the cooling channel. The small step at about $45 \mathrm{~m}$ corresponds to the change in absorber window thickness.

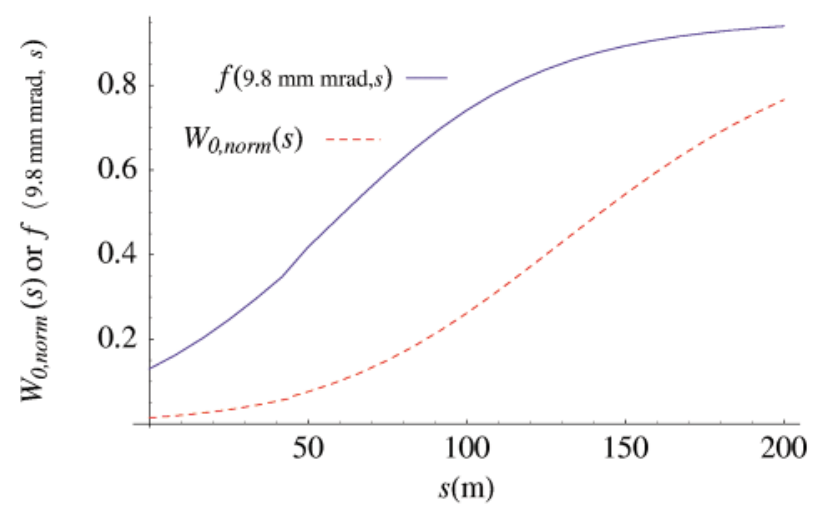

FIG. 6. (Color) Normalized peak density, as defined in Eq. (13.2), and fraction of the distribution within $9.8 \mathrm{~mm} \mathrm{rad}$ (geometric four-dimensional emittance), from Eq. (10.19), as a function of distance along the channel. expansion, and neglecting the nonlinear term in the last line of Eq. (4.12)] indicate even smaller errors. Thus it is not particularly surprising that the linear theory discussed here agrees well with the results of numerical simulations, for the transverse dynamics.

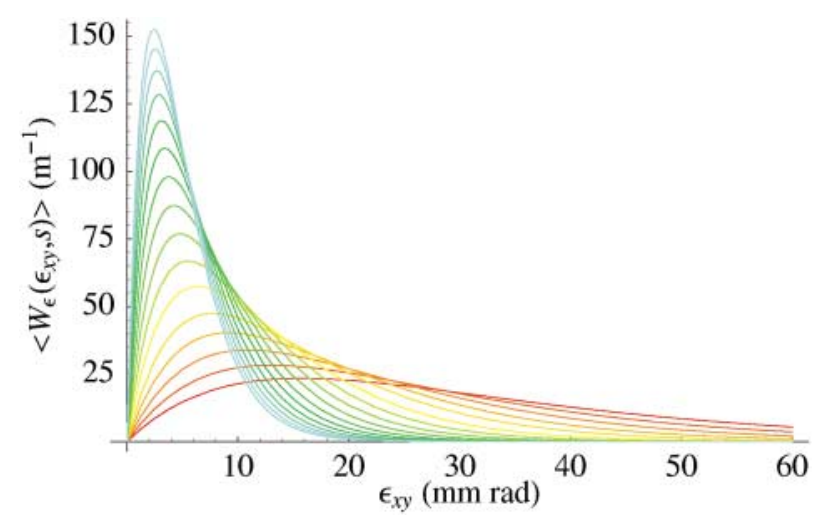

FIG. 7. (Color) Density distribution in the $\varepsilon_{x y}$ variable, at intervals of five periods along the channel, from period 0 to period 75. The plots are color coded according to the spectrum, varying from red to blue as the period number varies from 0 to 75 .

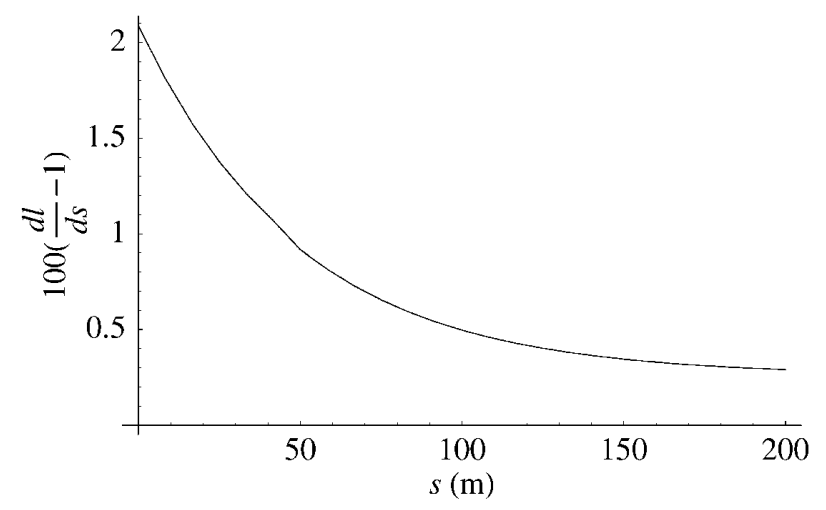

FIG. 8. An estimate of the error made by the paraxial approximation. 


\section{CONCLUSION}

This paper outlined a formalism for the description of the linear transverse dynamics of charged particles in an axisymmetric ionization cooling channel. The particle trajectories in the absence of Coulomb scattering were described in terms of lattice functions à la Courant and Snyder, which depend only on the electric and magnetic fields in the channel. The process of multiple Coulomb scattering, which introduces stochastic terms into the particle equations of motion, was treated (in Gaussian approximation) by obtaining the distribution function in phase space as a solution of a Fokker-Planck equation.

The distribution function was then used to obtain moment equations for the transverse variables, and for combinations of variables such as the emittance and angular momentum. The distribution function was also used to obtain an expression for the peak four-dimensional phase space density, and for the fraction of the beam that is within a certain area in phase space; these are important figures of merit for the design of cooling channels. The special, but very common, case of a periodic channel was then considered, and expressions for the asymptotic rms emittance and peak phase space density were obtained.

The application of the general formalism to a numerical example, based on the design of a cooling channel for a neutrino source, was considered, and comparisons were made with numerical simulations [8] of that channel. Finally, the relation of this work to a previous treatment [3] of transverse ionization cooling in the linear approximation was made.

Although numerical simulations of cooling channels will always be necessary, the formalism described here provides an alternative and complementary approach to the design and analysis of such channels. It may also provide the basis for further analytical work; for example, the effects of the (small) nonlinear terms neglected in Sec. IV could be treated perturbatively, with the linear analysis presented here as the zero-order approximation. Furthermore, if an analytic linear description of the longitudinal dynamics is developed, the work presented here could be used in conjunction with it to study the interplay between the longitudinal and transverse dynamics.

\section{ACKNOWLEDGMENT}

This work was supported by the National Science Foundation.

[1] A. N. Skrinsky and V. V. Parkhomchuk, Sov. J. Part. Nucl. 12, 223 (1981).

[2] D. Neuffer, Part. Accel. 14, 75 (1983).

[3] K. J. Kim and C. Wang, Phys. Rev. Lett. 85, 760 (2000).

[4] G. Penn and J. Wurtele, Phys. Rev. Lett. 85, 764 (2000).

[5] R. C. Fernow and J.C. Gallardo, Phys. Rev. E 52, 1039 (1995).

[6] E. D. Courant and H. S. Snyder, Ann. Phys. (Paris) 3, 1 (1958).

[7] S. Chandrasekar, Rev. Mod. Phys. 15, 1 (1943).

[8] A Feasibility Study of a Neutrino Source Based on a Muon Storage Ring, edited by N. Holtkamp and D. Finley (Fermilab, Batavia, IL, 2000).

[9] G. R. Lynch and O. I. Dahl, Nucl. Instrum. Methods Phys. Res., Sect. B 58, 6 (1991).

[10] John Jowett, in Physics of Particle Accelerators, edited by M. Month and M. Deines, AIP Conf. Proc. No. 153 (AIP, New York, 1987), p. 866.

[11] Problems in Probability Theory, Mathematical Statistics and the Theory of Random Functions, edited by A. A. Sveshnikov (Dover, New York, 1968), p. 92.

[12] C. Wang and K. J. Kim, Phys. Rev. E 63, 056502 (2001). 